\title{
EXISTENCE OF SOLUTIONS TO WEAK PARABOLIC EQUATIONS FOR MEASURES
}

\author{
VLADIMIR I. BOGACHEV, GIUSEPPE DA PRATO, AND MICHAEL RÖCKNER
}

\begin{abstract}
Let $A=\left(a^{i j}\right)$ be a Borel mapping on $[0,1] \times \mathbb{R}^{d}$ with values in the space of nonnegative operators on $\mathbb{R}^{d}$ and let $b=\left(b^{i}\right)$ be a Borel mapping on $[0,1] \times \mathbb{R}^{d}$ with values in $\mathbb{R}^{d}$. Let $L u(t, x)=\partial_{t} u(t, x)+a^{i j}(t, x) \partial_{x_{i}} \partial_{x_{j}} u(t, x)+b^{i}(t, x) \partial_{x_{i}} u(t, x), u \in C_{0}^{\infty}\left((0,1) \times \mathbb{R}^{d}\right)$. Under broad assumptions on $A$ and $b$, we construct a family $\mu=\left(\mu_{t}\right)_{t \in[0,1)}$ of probability measures $\mu_{t}$ on $\mathbb{R}^{d}$ which solves the Cauchy problem $L^{*} \mu=0$ with initial condition $\mu_{0}=\nu$, where $\nu$ is a probability measure on $\mathbb{R}^{d}$, in the following weak sense:

and

$$
\int_{0}^{1} \int_{\mathbb{R}^{d}} L u(t, x) \mu_{t}(d x) d t=0, \quad u \in C_{0}^{\infty}\left((0,1) \times \mathbb{R}^{d}\right),
$$

$$
\lim _{t \rightarrow 0} \int_{\mathbb{R}^{d}} \zeta(x) \mu_{t}(d x)=\int_{\mathbb{R}^{d}} \zeta(x) \nu(d x), \quad \zeta \in C_{0}^{\infty}\left(\mathbb{R}^{d}\right) .
$$

Such an equation is satisfied by transition probabilities of a diffusion process associated with $A$ and $b$ provided such a process exists. However, we do not assume the existence of a process and allow quite singular coefficients, in particular, $b$ may be locally unbounded or $A$ may be degenerate. An infinite dimensional analogue is discussed as well. Main methods are $L^{p}$-analysis with respect to suitably chosen measures and reduction to the elliptic case (studied previously) by piecewise constant approximations in time.
\end{abstract}

AMS Subject Classification: 35K10, 35K12, 60J35, 60J60, 47D07

\section{INTRODUCTION}

According to Kolmogorov's classical result, the transition probabilities of the diffusion process in $\mathbb{R}^{d}$ governed by the stochastic differential equation

$$
d \xi_{t}=\sigma\left(t, \xi_{t}\right) d W_{t}+b\left(t, \xi_{t}\right) d t
$$

with time-dependent coefficients satisfy the parabolic equation

$$
L^{*} p=0,
$$

where $A=\left(a^{i j}\right), A=\sigma^{2} / 2$, and $L^{*}$ is the formal adjoint to the operator

$$
L u(t, x):=\frac{\partial u(t, x)}{\partial t}+a^{i j}(t, x) \partial_{x_{i}} \partial_{x_{j}} u(t, x)+b^{i}(t, x) \partial_{x_{i}} u(t, x) .
$$

In the case of uniformly bounded coefficients and classical solutions, the above mentioned parabolic equation for probability measures was investigated in the important paper by Il'in and Hasminskii [8], where the main objective was to study the behaviour of solutions and their stabilization as $t \rightarrow \infty$. In recent years, there has been a growing interest in such equations for measures (defined in the weak sense as explained below) in the situation, where it is not assumed in advance that there exists a corresponding diffusion and if $a^{i j}$ and $b^{i}$ are not regular. In [5], families of measures satisfying equations of the above type have been studied in connection with flows of probability measures. An approach to constructing generalized diffusions via solutions to weak parabolic equations has been recently developed by W. Stannat [15]. However, in this paper, we do not address probabilistic issues (which 
will be the subject of another work) and consider only analytic problems, although these problems still keep a certain probabilistic flavour (in particular, we are interested in solutions that are probability measures). If the elliptic part $H$ of $L$ is time independent and there exists a probability measure $\mu$ on $\mathbb{R}^{d}$ such that $H^{*} \mu=0$ in the sense that

$$
\int_{\mathbb{R}^{d}} H \varphi d \mu=0 \quad \forall \varphi \in C_{0}^{\infty}\left(\mathbb{R}^{d}\right)
$$

then, under broad assumptions (involving the existence of a suitable Lyapunov function), there exists a strongly continuous Markov semigroup $\left(T_{t}\right)_{t \geq 0}$ on $L^{1}(\mu)$ (actually, on all $L^{p}(\mu)$, $p \in[1, \infty)$ ) whose generator coincides with $H$ on $C_{0}^{\infty}\left(\mathbb{R}^{d}\right)$ and which serves as the transition semigroup of a diffusion process (see [14]). As shown in [1, Section 4], the adjoint semigroup $\left(T_{t}^{*}\right)_{t \geq 0}$ can be extended even to bounded measures on $\mathbb{R}^{d}$ and, for any probability measure $\nu$ on $\mathbb{R}^{d}$, the family $\left(T_{t}^{*} \nu\right)_{t \geq 0}$ consists of probability measures with densities $p(t, x)$ and the measure $\mu=T_{t}^{*} \nu d t$ on $[0,1) \times \mathbb{R}^{d}$ satisfies the weak parabolic equation $L^{*} \mu=0$. In this work, we investigate the existence problem in the time-dependent case. The problem is as follows.

Let $A(t, x)=\left(a^{i j}(t, x)\right)_{1 \leq i, j \leq d}$ be a Borel mapping on $[0,1] \times \mathbb{R}^{d}$ with values in the space of nonnegative operators on $\mathbb{R}^{d}$ and let $b(t, x)=\left(b^{i}(t, x)\right)$ be a Borel mapping on $[0,1] \times \mathbb{R}^{d}$ with values in $\mathbb{R}^{d}$. Let

$$
L u(t, x):=\frac{\partial u(t, x)}{\partial t}+a^{i j}(t, x) \partial_{x_{i}} \partial_{x_{j}} u(t, x)+b^{i}(t, x) \partial_{x_{i}} u(t, x)
$$

for $u \in C_{0}^{\infty}\left((0,1) \times \mathbb{R}^{d}\right)$. The elliptic part of $L$ is denoted by

$$
H u(t, x):=a^{i j}(t, x) \partial_{x_{i}} \partial_{x_{j}} u(t, x)+b^{i}(t, x) \partial_{x_{i}} u(t, x) .
$$

We shall say that a family of Radon measures $\mu=\left(\mu_{t}\right)_{t \in[0,1)}$ on $\mathbb{R}^{d}$ satisfies the weak parabolic equation

$$
L^{*} \mu=0
$$

if the functions $a^{i j}$ and $b^{i}$ are integrable on every compact set in $(0,1) \times \mathbb{R}^{d}$ with respect to the measure $\mu_{t} d t$ and, for every $u \in C_{0}^{\infty}\left((0,1) \times \mathbb{R}^{d}\right)$, one has

$$
\int_{0}^{1} \int_{\mathbb{R}^{d}} L u(t, x) \mu_{t}(d x) d t=0 .
$$

We shall say that $\mu$ satisfies the initial condition $\mu_{0}:=\nu$ at $t=0$ if $\nu$ is a measure on $\mathbb{R}^{d}$ and

$$
\lim _{t \rightarrow 0} \int_{\mathbb{R}^{d}} \zeta(x) \mu_{t}(d x)=\int_{\mathbb{R}^{d}} \zeta(x) \nu(d x)
$$

for all $\zeta \in C_{0}^{\infty}\left(\mathbb{R}^{d}\right)$. In this case we write $\mu=\left(\mu_{t}\right)_{t \in[0,1)}$.

Our principal goal is to construct solutions to such equations so that $\mu_{t}$ is a probability measure for every $t \in[0,1)$ (in finite and infinite dimensions) under the assumption that there exists a suitable Lyapunov function. The corresponding results generalize those obtained in [2] in the elliptic case (however, the latter are used here). It turns out that the existence of solutions can be proved under quite general assumptions, in particular, without any regularity of $a^{i j}$ and $b^{i}$. In contrast to the case of classical weak solutions, such solutions may be extremely irregular. For example, if $d=1, b=0, a(t, x)=\alpha(x)$ is an arbitrary positive measurable function such that $1 / \alpha$ is integrable, then the family of measures $\mu_{t} \equiv \alpha^{-1} d x$ solves the corresponding problem. Our approach to constructing solutions is based on establishing certain a priori estimates of two types: global, which involve suitable Lyapunov functions and yield uniform tightness, and local, which yield uniform boundedness 
of $L^{p}$-norms on compact sets and enable us to verify (1.3) for measures constructed by means of tightness.

In the case when $A$ and $b$ are independent of $t$ and satisfy the hypotheses of Theorem 3.1 below, our solution coincides with the aforementioned family $\left(T_{t}^{*} \nu\right)_{t \in[0,1)}$. In general, under the hypotheses of Theorem 3.1, all possible solutions possess certain local differentiability properties (in particular, the density $p_{t}$ of $\mu_{t}$ belongs to the Sobolev class $H_{l o c}^{p, 1}\left(\mathbb{R}^{d}\right)$ ), which enables one to write equation (1.3) in the classical weak form

$$
\int_{0}^{1} \int_{\mathbb{R}^{d}}\left[\frac{\partial u}{\partial t} p_{t}-a^{i j} \partial_{x_{i}} p_{t} \partial_{x_{j}} u-p_{t} \partial_{x_{i}} a^{i j} \partial_{x_{j}} u+p_{t} b^{i} \partial_{x_{i}} u\right] d x d t=0 .
$$

However, typically the functions $p_{t}$ are neither bounded nor square integrable over $\mathbb{R}^{d}$ with respect to Lebesgue measure, i.e., do not belong to the functional classes considered, e.g., in [7], [11], [12], [13]. If the coefficients $a^{i j}$ are not differentiable (as in the corollaries to the main theorem), then the functions $p_{t}$ may not be differentiable as well (in the case of degenerate $A$ they even need not exist). As shown in [1], for nondegerate $A$, the differentiability properties of $p_{t}$ are essentially the same as those of $a^{i j}$ and simple examples show that they may be not better.

The main novelty of the method in this paper is first to use extensively $L^{p}$-analysis with respect to suitably chosen measures (different from Lebesgue measure) which are deeply related to the coefficients of the given operator. Second, a piecewise constant approximation enables us to use our previously obtained results in the elliptic case.

\section{AuXiliary RESUlts}

Let us recall some standard notation for various Sobolev classes on $\mathbb{R}^{d}$ or on open sets $U \subset \mathbb{R}^{d}$. The class $H^{p, 1}(U)$ consists of all functions $f \in L^{p}(U)$ with generalized partial derivatives $\partial_{x_{i}} f \in L^{p}(U)$. This space is equipped with its natural Sobolev norm $\|f\|_{p, 1}$. Let $H_{0}^{p, 1}(U)$ be the closure in $H^{p, 1}(U)$ of $C_{0}^{\infty}(U)$ (the class of infinitely differentiable functions with compact support in $U)$. For a function $u$ on $(0,1) \times \mathbb{R}^{d}$, we set $\partial_{t} u(t, x):=\partial u(t, x) / \partial t$.

Let $B$ be an open ball in $\mathbb{R}^{d}$. Set $S:=([0,1) \times \partial B) \cup(\{0\} \times B)$. The space $\mathbb{H}^{p, 1}([0,1], B)$ consists of all measurable functions $w$ on $[0,1] \times B$ such that, for each $t \in[0,1)$, one has $w(t, \cdot) \in H^{p, 1}(B)$ and

$$
\|w\|_{\mathbb{H}^{p, 1}([0,1], B)}:=\left(\int_{0}^{1}\|w(t, \cdot)\|_{p, 1}^{p} d t\right)^{1 / p}<\infty .
$$

The space $\mathbb{H}_{0}^{p, 1}([0,1], B)$ is the subspace in $\mathbb{H}^{p, 1}([0,1], B)$ formed by the functions with $w(t, \cdot) \in H_{0}^{p, 1}(B)$ for all $t \in[0,1)$. For convenience of notation all functions defined on $[0,1) \times \mathbb{R}^{d}$ will be considered also as functions on $[0,1] \times \mathbb{R}^{d}$.

Lemma 2.1. If $\mu=\left(\mu_{t}\right)_{t \in[0,1)}$ satisfies (1.3) and (1.5), then for almost all $t \in[0,1)$ one has

$$
\int_{\mathbb{R}^{d}} \zeta(x) \mu_{t}(d x)-\int_{0}^{t} \int_{\mathbb{R}^{d}} L \zeta(s, x) \mu_{s}(d x) d s=\int_{\mathbb{R}^{d}} \zeta(x) \nu(d x) \quad \text { for every } \zeta \in C_{0}^{\infty}\left(\mathbb{R}^{d}\right) .
$$

If, for each $\zeta \in C_{0}^{\infty}\left(\mathbb{R}^{d}\right)$, the function $\int_{\mathbb{R}^{d}} \zeta(x) \mu_{t}(d x)$ is continuous on $[0,1)$, then $(2.1)$ holds for all $t \in[0,1)$ and is equivalent to (1.3) and (1.5).

Proof. Let $u(t, x)=\varphi(t) \zeta(x)$, where $\varphi \in C_{0}^{\infty}(0,1)$ and $\zeta \in C_{0}^{\infty}\left(\mathbb{R}^{d}\right)$, and let

$$
f(t):=\int_{\mathbb{R}^{d}} \zeta(x) \mu_{t}(d x), \quad h(t):=\int_{\mathbb{R}^{d}} L \zeta(t, x) \mu_{t}(d x) .
$$


Then we derive from (1.3), where we use the identity $\partial \zeta / \partial t=0$, that $f^{\prime}=h$ in the sense of distributions on $(0,1)$. Taking into account $(1.5)$ we arrive at $(2.1)$, where, however, the full measure set in $t$ may depend on $\zeta$. Hence such a set can be chosen common for any given countable set of functions $\zeta_{j}$. This enables one to find a common full measure set in $(0,1)$ for all $\zeta \in C_{0}^{\infty}\left(\mathbb{R}^{d}\right)$, since there exists a countable family of functions $\zeta_{j} \in C_{0}^{\infty}\left(\mathbb{R}^{d}\right)$ such that every function $\zeta \in C_{0}^{\infty}\left(\mathbb{R}^{d}\right)$ can be approximated uniformly with the first and second derivatives by a sequence of functions in $\left\{\zeta_{j}\right\}$ with support in a common ball. Certainly, if $\int \zeta(x) \mu_{t}(d x)$ is continuous in $t$, then we obtain the above equality for all $t$. The converse is also clear.

It is worth noting that the reason why we require that all measures $\mu_{t}$ (and not just almost all) in the next lemma be probabilities is that this is the case when one deals with transition probabilities. From the analytical point of view, this is not essential, of course.

Lemma 2.2. Let $\mu=\left(\mu_{t}\right)_{t \in[0,1)}$ be a family of probability measures on $\mathbb{R}^{d}$ satisfying (1.3) and (1.5), where $\nu$ is a probability measure on $\mathbb{R}^{d}$. Suppose that there exists a nonnegative function $\Psi \in C^{2}\left(\mathbb{R}^{d}\right)$ such that $\Psi \in L^{1}(\nu), \lim _{|x| \rightarrow \infty} \Psi(x)=+\infty$, and

$$
L \Psi(t, x) \leq C \quad \mu_{t} d t \text {-a.e. }
$$

with some constant $C \geq 0$. Then

$$
\int_{\mathbb{R}^{d}} \Psi(x) \mu_{t}(d x) \leq C t+\int_{\mathbb{R}^{d}} \Psi(x) \nu(d x)
$$

for a.e. $t \in[0,1)$. If the functions $t \mapsto \int_{\mathbb{R}^{d}} \zeta(x) \mu_{t}(d x), \zeta \in C_{0}^{\infty}\left(\mathbb{R}^{d}\right)$, are continuous on $[0,1)$, then $(2.3)$ is true for all $t \in[0,1)$.

Proof. It is clear that (2.1) remains true also for $\zeta \in C_{b}^{\infty}\left(\mathbb{R}^{d}\right)$ such that $\zeta(x)=q=$ const outside some ball. Indeed, the function $\zeta_{0}:=\zeta(x)-q$ is of compact support, $L \zeta_{0}=L \zeta$ and

$$
\int_{\mathbb{R}^{d}} q \mu_{t}(d x)=\int_{\mathbb{R}^{d}} q \nu(d x)=q .
$$

Furthermore, due to the local integrability of the functions $a^{i j}$ and $b^{i}$ with respect to $\mu_{t} d t$, (2.1) is clearly still true for $\zeta \in C_{b}^{2}\left(\mathbb{R}^{d}\right)$ (in place of $C_{0}^{\infty}$ ) such that $\zeta(x)=q=$ const outside some ball. Now let us fix $k \in \mathbb{N}$ and take a function $\theta_{k} \in C^{2}(\mathbb{R})$ such that $\theta_{k}(r)=r$ if $r \leq k$, $\theta_{k}(r)=k+1$ if $r \geq k+2,0 \leq \theta_{k}^{\prime}(r) \leq 1$, and $\theta_{k}^{\prime \prime}(r) \leq 0$. By our assumption on $\Psi, \theta_{k} \circ \Psi$ is constant outside a sufficiently large ball. Hence, as explained above, (2.1) is true with $\zeta(x)=\zeta_{k}(x):=\theta_{k}(\Psi(x))$. We observe that

$$
L \zeta_{k}=\theta_{k}^{\prime}(\Psi) L \Psi+\theta_{k}^{\prime \prime}(\Psi)\langle A \nabla \Psi, \nabla \Psi\rangle \leq \theta_{k}^{\prime}(\Psi) L \Psi
$$

Therefore, for a.e. $t \in[0,1)$

$$
\begin{aligned}
\int_{\mathbb{R}^{d}} \theta_{k} \circ \Psi d \mu_{t} & =\int_{\mathbb{R}^{d}} \theta_{k} \circ \Psi d \nu+\int_{0}^{t} \int_{\mathbb{R}^{d}} L \zeta_{k} d \mu_{s} d s \\
& \leq \int_{\mathbb{R}^{d}} \theta_{k} \circ \Psi d \nu+\int_{0}^{t} \int_{\mathbb{R}^{d}}\left(\theta_{k}^{\prime} \circ \Psi\right) L \Psi d \mu_{s} d s \\
& \leq \int_{\mathbb{R}^{d}} \Psi d \nu+C t
\end{aligned}
$$

By Fatou's lemma we arrive at the estimate

$$
\int_{\mathbb{R}^{d}} \Psi d \mu_{t} \leq \int_{\mathbb{R}^{d}} \Psi d \nu+C t .
$$


Corollary 2.3. Let $\left(\mu_{t}\right)_{t \in[0,1)}$ be as in Lemma 2.2 and let $\Psi(x):=|x|^{2}$. Suppose that $L \Psi(t, x) \leq C$ and trace $A(t, x) \leq C^{\prime}+C^{\prime \prime} \Psi(x) \quad \mu_{t} d t$-a.e. for some nonnegative constants $C, C^{\prime}$ and $C^{\prime \prime}$, and that $\nu$ has all moments. Then, for every $m \in \mathbb{N}$, there exists $C_{m}$, which depends only on $m, C$, and $\int_{\mathbb{R}^{d}}|x|^{2 m} \nu(d x)$, such that

$$
\int_{\mathbb{R}^{d}}|x|^{2 m} \mu_{t}(d t) \leq C_{m}
$$

for a.e. $t \in[0,1)$. If the functions $t \mapsto \int_{\mathbb{R}^{d}} \zeta(x) \mu_{t}(d x), \zeta \in C_{0}^{\infty}\left(\mathbb{R}^{d}\right)$, are continuous on $[0,1)$, then $(2.4)$ is true for all $t \in[0,1)$.

Proof. If $m=1$ we can take $C_{1}:=C+\int_{\mathbb{R}^{d}}|x|^{2} \nu(d x)$ according to Lemma 2.2. Suppose that our claim is true for some $m \in \mathbb{N}$. Let us consider the function $\Psi_{m+1}(x):=|x|^{2 m+2}$. Then we obtain

$$
\begin{aligned}
L \Psi_{m+1}(t, x) & =4 m(m+1) \Psi_{m-1}(x)\langle A(t, x) x, x\rangle+2(m+1) \Psi_{m}(x) L \Psi(t, x) \\
& \leq \kappa_{1} \Psi_{m}+\kappa_{2} \Psi_{m+1}
\end{aligned}
$$

where

$$
\kappa_{1}:=2 C(m+1)+4 C^{\prime} m(m+1), \kappa_{2}:=4 C^{\prime \prime} m(m+1) .
$$

Now the same reasoning as in the proof of the above lemma yields the estimate

$$
\begin{aligned}
\int_{\mathbb{R}^{d}} \theta_{k} \circ \Psi_{m+1} d \mu_{t} & \leq \int_{\mathbb{R}^{d}} \Psi_{m+1} d \nu+\int_{0}^{t} \int_{\mathbb{R}^{d}} \theta_{k}^{\prime}\left(\Psi_{m+1}\right)\left(\kappa_{1} \Psi_{m}+\kappa_{2} \Psi_{m+1}\right) d \mu_{s} d s \\
& \leq \int_{\mathbb{R}^{d}} \Psi_{m+1} d \nu+\kappa_{1} C_{m}+\kappa_{2} \int_{0}^{t} \int_{\mathbb{R}^{d}} \theta_{k}^{\prime}\left(\Psi_{m+1}\right) \Psi_{m+1} d \mu_{s} d s,
\end{aligned}
$$

where the last step follows by the hypothesis of induction. Let

$$
f_{k}(t):=\int_{\mathbb{R}^{d}} \theta_{k} \circ \Psi_{m+1} d \mu_{t}
$$

We observe that $\theta_{k}^{\prime}(t) t \leq \frac{k+2}{k} \theta_{k}(t)$. Indeed, if $t<k$, then $\theta_{k}(t)=t$ and $\theta_{k}^{\prime}(t)=1$, if $t>k+2$, then $\theta_{k}^{\prime}(t)=0$, and if $k \leq t \leq k+2$, then $\theta_{k}(t) \geq k$ and $\theta_{k}^{\prime}(t) \leq 1$. Hence $\theta_{k}^{\prime}\left(\Psi_{m+1}\right) \Psi_{m+1} \leq \frac{k+2}{k} \theta_{k}\left(\Psi_{m+1}\right)$ and the above estimate yields that

$$
f_{k}(t) \leq C_{m}^{\prime}+\kappa_{2} \frac{k+2}{k} \int_{0}^{t} f_{k}(s) d s
$$

where $C_{m}^{\prime}$ is some constant. By Gronwall's inequality we obtain

$$
f_{k}(t) \leq C_{m}^{\prime} \exp \left(\kappa_{2} \frac{k+2}{k} t\right) .
$$

Letting $k \rightarrow \infty$, we arrive at the desired conclusion by Fatou's lemma.

Corollary 2.4. Let $\mu=\left(\mu_{t}\right)_{t \in[0,1)}$ be a family of probability measures on $\mathbb{R}^{d}$ satisfying (1.3) and (1.5), where $\nu$ is a probability measure on $\mathbb{R}^{d}$. Let $\Psi \in C^{2}\left(\mathbb{R}^{d}\right)$ be a nonnegative function such that $\lim _{|x| \rightarrow \infty} \Psi(x)=+\infty$ and $L \Psi \leq C \mu_{t} d t$-a.e., where $C \geq 0$ is a constant. Then one can find a nonnegative function $\Psi_{0} \in C^{2}\left(\mathbb{R}^{d}\right)$ such that $\lim _{|x| \rightarrow \infty} \Psi_{0}(x)=+\infty, L \Psi_{0} \leq C \mu_{t} d t$-a.e. and $\Psi_{0} \in L^{1}(\nu)$. 
Moreover, if $\mathfrak{M}$ is a uniformly tight family of probability measures on $\mathbb{R}^{d}$ and for every $\nu \in$ $\mathfrak{M}$ there exists a solution $\mu^{\nu}=\left(\mu_{t}^{\nu}\right)_{t \in[0,1)}$ of the problem (1.3), (1.5) with initial condition $\nu$, then one can find a function $\Psi_{0}$ as above such that

$$
\sup \left\{\int_{\mathbb{R}^{d}} \Psi_{0} d \mu_{t}^{\nu}, \nu \in \mathfrak{M}, t \in[0,1)\right\} \leq \sup \left\{C+\int_{\mathbb{R}^{d}} \Psi_{0} d \nu, \nu \in \mathfrak{M}\right\}<\infty .
$$

Proof. Indeed, one can find a function $\theta \in C^{2}(\mathbb{R})$ nonnegative on $\mathbb{R}_{+}$such that $\Psi_{0}:=\theta \circ \Psi \in$ $L^{1}(\nu), \lim _{r \rightarrow+\infty} \theta(r)=+\infty, 0 \leq \theta^{\prime} \leq 1$, and $\theta^{\prime \prime} \leq 0$. Then $L \Psi_{0} \leq \theta^{\prime} \circ \Psi L \Psi \leq C$. Moreover, for any uniformly tight family of probability measures $\mathfrak{M}$, such a function $\theta$ can be found common for all $\nu \in \mathfrak{M}$ with $\sup _{\nu \in \mathfrak{M}} \int_{\mathbb{R}^{d}} \Psi_{0} d \nu<\infty$.

Remark 2.5. It is obvious from the proof that the condition $L \Psi \leq C$ in Lemma 2.2 can be relaxed as follows: there exists a measurable set $E \subset \mathbb{R}^{d}$ such that $L \Psi(t, x) \leq C \mu_{t} d t$-a.e. on $(0,1) \times\left(\mathbb{R}^{d} \backslash E\right)$ and $C^{\prime}:=\int_{0}^{1} \int_{E}|L \Psi(t, x)| \mu_{t}(d x) d t<\infty$. Then in the left-hand side of (2.3) one should add $C^{\prime}$.

It is also worth mentioning that if in the situation of Lemma 2.2 the functions $a^{i j}$ are bounded on bounded subsets of $[0,1] \times \mathbb{R}^{d}$ and (2.2) holds only for $x$ outside some bounded set, then one can find another nonnegative function $\Psi_{0} \in C^{2}\left(\mathbb{R}^{d}\right)$ such that $\Psi_{0}$ coincides with $\Psi$ outside some bounded set and $L \Psi_{0}(t, x) \leq C_{0} \mu_{t} d t$-a.e., where $C_{0}$ is a positive constant. Indeed, let $\theta \in C^{\infty}\left(\mathbb{R}^{1}\right)$ be such that $\theta(s)=0$ if $s \leq-1, \theta(s)=s$ if $s \geq$ $1,0 \leq \theta^{\prime} \leq 1$. There exists $k$ such that $L \Psi \leq C \mu_{t} d t$-a.e. if $|x| \geq k$. Let $M:=$ $\sup _{s}\left|\theta^{\prime \prime}(s)\right| \sup _{t \in[0,1],|x| \leq k+2}\langle A(t, x) \nabla \Psi(x), \nabla \Psi(x)\rangle$ and take $\Psi_{0}(x):=\theta(\Psi(x)-k)$. Then

$L \Psi_{0}(t, x)=\theta^{\prime}(\Psi(x)-k-1) L \Psi(t, x)+\theta^{\prime \prime}(\Psi(x)-k-1)\langle A(t, x) \nabla \Psi(x), \nabla \Psi(x)\rangle \leq C+M$, since $\theta^{\prime}(\Psi(x)-k-1)=0$ if $|x| \leq k, L \Psi(x) \leq C$ if $|x| \geq k$, and $\theta^{\prime \prime}(\Psi(x)-k-1)=0$ if $|x| \leq k+2$.

Let us introduce the following conditions on $A, b, p \in[1,+\infty)$, and an open ball $B \subset \mathbb{R}^{d}$ : (C1) there exist two constants $M_{1}=M_{1}(B)$ and $M_{2}(B)$ such that for all $i, j$ one has

$$
\operatorname{det}_{(t, x) \in[0,1] \times B} A(t, x) \geq M_{1} \text { and } \sup _{t \in[0,1]}\left\|a^{i j}(t, \cdot)\right\|_{H^{p, 1}(B)} \leq M_{2} .
$$

(C2) there exists $M_{3}=M_{3}(B)$ such that for all $i$ one has

$$
\sup _{t \in[0,1]}\left\|b^{i}(t, \cdot)\right\|_{L^{p}(B)} \leq M_{3} .
$$

It follows from (C1) and the Sobolev embedding theorem that if $p>d$, then every functions $a^{i j}$ has a jointly measurable version such that all functions $x \mapsto a^{i j}(t, x), t \in(0,1)$, are Hölder continuous of order $1-d / p$ and bounded on $B$ uniformly in $t$ (their Hölder and sup-norms on $B$ are estimated by a constant depending on $p, d, B$ and $M_{2}$ ). Below we use the same notation $a^{i j}$ for these particular versions.

We need a technical result on weak solutions of the equation

$$
\partial_{t} w-a^{i j} \partial_{x_{i}} \partial_{x_{j}} w=\partial_{x_{i}} h^{i}
$$

with zero boundary condition on $S:=([0,1) \times \partial B) \cup(\{0\} \times B)$, where $h^{i} \in L^{p}((0,1) \times B)$. A function $w$ on $[0,1) \times \mathbb{R}^{d}$ is said to be a solution of $(2.5)$ with zero boundary condition if

(i) $w$ is continuous and belongs to the space $\mathbb{H}_{0}^{p, 1}([0,1], B)$, 
(ii) for every function $\varphi \in C_{0}^{\infty}\left(\mathbb{R}^{1} \times \mathbb{R}^{d}\right)$ with support in $[-1,1) \times B$, one has

$$
\int_{0}^{1} \int_{\mathbb{R}^{d}}\left[-w \partial_{t} \varphi+a^{i j} \partial_{x_{i}} w \partial_{x_{j}} \varphi+\varphi \partial_{x_{i}} a^{i j} \partial_{x_{j}} w\right] d x d t=-\int_{0}^{1} \int_{\mathbb{R}^{d}} h^{i} \partial_{x_{i}} \varphi d x d t .
$$

Note that (2.6) implies also that, for every $\xi \in C_{0}^{\infty}(B)$, one has

$$
\int_{\mathbb{R}^{d}} w(0, x) \xi(x) d x=0,
$$

which expresses the zero boundary value at $t=0$, whereas the zero boundary value on $[0,1] \times \partial B$ is taken care of by the condition $w(t, \cdot) \in H_{0}^{p, 1}(B)$. Indeed, let

$$
f(t):=\int_{\mathbb{R}^{d}} w(t, x) \xi(x) d x, \quad g(t):=\int_{\mathbb{R}^{d}}\left[a^{i j} \partial_{x_{i}} w \partial_{x_{j}} \xi+\xi \partial_{x_{i}} a^{i j} \partial_{x_{j}} w+h^{i} \partial_{x_{i}} \xi\right] d x .
$$

Then, for every $\psi \in C_{0}^{\infty}\left(\mathbb{R}^{1}\right)$ with support in $[-1,1)$, we have

$$
\int_{0}^{1} \psi^{\prime}(t) f(t) d t=\int_{0}^{1} \psi(t) g(t) d t .
$$

Hence $f^{\prime}=-g$ in the sense of distributions. Therefore, $f$ is absolutely continuous and there is a constant $C$ such that $f(t)=C-\int_{0}^{t} g(s) d s$, which yields $C=0$.

Let $p>d+2$. Suppose that there exist two constants $M_{1}$ and $M_{2}$ (which depend only on $A$ and $B$ ) such that $\operatorname{det} A(t, x) \geq M_{1}$ and $\sup \left\|a^{i j}(t, \cdot)\right\|_{H^{p, 1}(B)} \leq M_{2}$. Then, it follows from the proof of [1, Lemma 3.5] that there exist $R>0$ and $M>0$ (which depend only on $B, d$, $p, M_{1}$, and $M_{2}$ ) such that, for every open ball $B_{R}$ of radius $R$ in $B$ and any solution $w$ of equation (2.5) with zero boundary condition on $\left([0,1) \times \partial B_{R}\right) \cup\left(\{0\} \times B_{R}\right)$ one has

$$
\|w\|_{\mathbb{H}^{p, 1}\left([0,1], B_{R}\right)} \leq M\left\|\left(h^{i}\right)\right\|_{L^{p}\left((0,1) \times B_{R}\right)} .
$$

In a similar manner, for $b^{i} \in L^{p}([0,1) \times B)$, we say that a continuous function $w$ on $[0,1) \times \mathbb{R}^{d}$ satisfies the equation

$$
\partial_{t} w-a^{i j} \partial_{x_{i}} \partial_{x_{j}} w-b^{i} \partial_{x_{i}} w=0, \quad w(0, x)=\zeta(x),
$$

on $[0,1) \times \mathbb{R}^{d}$, where $b^{i} \in L^{p}\left([0,1) \times \mathbb{R}^{d}\right)$ and $\zeta \in C_{0}^{\infty}\left(\mathbb{R}^{d}\right)$, if, for every ball $B \subset \mathbb{R}^{d}, w$ belongs to the space $\mathbb{H}^{p, 1}([0,1], B)$, and, for every function $\varphi \in C_{0}^{\infty}\left(\mathbb{R}^{1} \times \mathbb{R}^{d}\right)$ with support in $[-1,1) \times \mathbb{R}^{d}$, one has

$$
\int_{0}^{1} \int_{\mathbb{R}^{d}}\left[-w \partial_{t} \varphi+a^{i j} \partial_{x_{i}} w \partial_{x_{j}} \varphi+\varphi \partial_{x_{i}} a^{i j} \partial_{x_{j}} w-\varphi b^{i} \partial_{x_{i}} w\right] d x d t=\int_{\mathbb{R}^{d}} \zeta(x) \varphi(0, x) d x
$$

One can verify that (2.9) indeed implies that, for every $\xi \in C_{0}^{\infty}\left(\mathbb{R}^{d}\right)$, one has $w(0, x)=\zeta(x)$.

Lemma 2.6. Suppose $w$ is a continuous function on $[0,1) \times \mathbb{R}^{d}$ satisfying $(2.8)$, where $\zeta \in$ $C_{0}^{\infty}\left(\mathbb{R}^{d}\right), a^{i j}(t, \cdot) \in H_{\text {loc }}^{p, 1}\left(\mathbb{R}^{d}\right), b^{i}(t, \cdot) \in L_{\text {loc }}^{p}\left(\mathbb{R}^{d}\right), t \in[0,1), p>d+2$, and for every centered ball $B_{R}$ of radius $R$ in $\mathbb{R}^{d}$, there exists a constant $C(R)$ such that

$$
\sup _{t \in[0,1)}\left[\left\|a^{i j}(t, \cdot)\right\|_{H^{p, 1}\left(B_{R}\right)}+\left\|b^{i}(t, \cdot)\right\|_{L^{p}\left(B_{R}\right)}+\inf _{x \in B_{R}} \operatorname{det} A^{-1}(t, x)\right] \leq C(R) .
$$

Suppose that $\sup |w| \leq 1$. Then, for all $R \in(0,1), x, y \in B_{R / 2}$ and all $t, s \in[0, R]$, one has

$$
|w(t, x)-w(s, y)| \leq M(R, C(R), T, d, p, \zeta)\left[|t-s|^{\gamma}+|x-y|^{\gamma}\right],
$$

where $M(R, C(R), d, p, \zeta)$ and $\gamma=\gamma(d, p)$ depend on the indicated objects only. 
Proof. Let us fix a function $\theta \in C_{0}^{\infty}\left(\mathbb{R}^{d}\right)$ such that $0 \leq \theta \leq 1,\left.\theta\right|_{B_{R / 2}}=1$, supp $\theta \subset B_{R}$. The function $v(t, x):=\theta(x) w(t, x)-\theta(x) \zeta(x)$ satisfies the equation

$$
\partial_{t} v(t, x)-a^{i j}(t, x) \partial_{x_{i}} \partial_{x_{j}} v(t, x)-b^{i} \partial_{x_{i}} v(t, x)=-h,
$$

where for $H$ as in (1.2) one has

$$
\begin{aligned}
h:=w H \theta+2 a^{i j} \partial_{x_{i}} w \partial_{x_{j}} \theta-H(\theta \zeta) & \\
& =w H \theta-H(\theta \zeta)-2 w \partial_{x_{i}}\left(a^{i j} \partial_{x_{j}} \theta\right)+2 \partial_{x_{i}}\left(w a^{i j} \partial_{x_{j}} \theta\right),
\end{aligned}
$$

with zero boundary condition on $\left([0,1] \times \partial B_{R}\right) \cup\left(\{0\} \times B_{R}\right)$. We shall prove that there exist functions $f^{i} \in L^{p}\left([0,1] \times B_{R}\right)$ such that

$$
\partial_{x_{i}} f^{i}=h
$$

and

$$
\left\|\left(f^{i}\right)\right\|_{L^{p}\left([0,1] \times B_{R}\right)} \leq K(R, C(R), d, p, \zeta, \theta),
$$

where $K(R, C(R), d, p, \zeta, \theta)$ depends only on the indicated objects. Indeed, for every fixed $t$, let $g(t, \cdot)$ be the solution of the Dirichlet problem

$$
\Delta g=w H \theta-H(\theta \zeta)-2 w \partial_{x_{i}} a^{i j} \partial_{x_{j}} \theta-2 w a^{i j} \partial_{x_{i}} \partial_{x_{j}} \theta, \quad x \in B_{R},
$$

with zero boundary condition on $\partial B_{R}$. Then we can set

$$
f^{i}(t, x):=\partial_{x_{i}} g(t, x)+2 w(t, x) a^{i j}(t, x) \partial_{x_{j}} \theta(x)
$$

and employ the fact that the $L^{p}$-norm of the gradient of the solution of the above Dirichlet problem is estimated via the $L^{p}$-norm of the right-hand side, which is majorized by

$$
\begin{aligned}
\left\|a^{i j}(t, \cdot)\right\|_{L^{p}\left(B_{R}\right)} \sup _{x \in B_{R}}\left[\left|\partial_{x_{i}} \partial_{x_{j}} \theta(x)\right|\right. & \left.+\left|\partial_{x_{i}} \partial_{x_{j}}(\theta \zeta)(x)\right|\right]+2\left\|\partial_{x_{i}} a^{i j}(t, \cdot)\right\|_{L^{p}\left(B_{R}\right)} \sup _{x \in B_{R}}\left|\partial_{x_{i}} \theta(x)\right| \\
& +\left\|b^{i}(t, \cdot)\right\|_{L^{p}\left(B_{R}\right)} \sup _{x \in B_{R}}\left[\left|\partial_{x_{i}} \theta(x)\right|+\left|\partial_{x_{i}}(\theta \zeta)(x)\right|\right] .
\end{aligned}
$$

Therefore, according to $(2.7)$ with $h^{i}:=b^{i}+f^{i}$, we majorize $\|w\|_{\mathbb{H}^{p, 1}\left([0,1], B_{R / 2}\right)}$ by a number, depending only on $R, C(R), d, p, \zeta$ and $\theta$ (but $\theta$ is fixed for each $R$ ). This yields (as in [1, Theorem 3.8] by an embedding theorem from [10]), the uniform boundedness of the Hölder norm of a certain order $\gamma$ (depending only on $d$ and $p$ ) of the function $w$ on $[0, R] \times B_{R}$.

We shall say that a function $V$ on $\mathbb{R}^{d}$ is compact if $\lim _{|x| \rightarrow+\infty} V(x)=+\infty$. We recall that if $A$ and $b$ do not depend on $t$ satisfy $(\mathrm{C} 1)$ and $(\mathrm{C} 2)$ with $p>d$ for every ball and if there is a compact function $V$ such that $\lim _{|x| \rightarrow \infty} H V(x)=-\infty$ (such a function is called a Lyapunov function), then there exists a unique probability measure $\mu$ on $\mathbb{R}^{d}$ satisfying the equation $H^{*} \mu=0$ (see [2], [3]). In addition, $\mu=\varrho d x$, where $\varrho \in H_{l o c}^{p, 1}\left(\mathbb{R}^{d}\right)$ is strictly positive and locally Hölder continuous. Finally, as shown by W. Stannat [14], there exists a unique strongly continuous semigroup $\left(T_{t}^{\mu}\right)_{t>0}$ on $L^{1}(\mu)$ whose generator extends $H$. This semigroup is Markovian and $\mu$ is its invariant measure and also a unique solution of $H^{*} \mu=0$ (see [3]). Moreover, if $p>d+2$, there is a semigroup of probability kernels $K_{t}(x, d y)=\varrho_{t}(x, y) d y$ such that for every $f \in L^{1}(\mu)$ the function

$$
K_{t}^{\mu} f(x):=\int_{\mathbb{R}^{d}} f(y) \varrho_{t}(x, y) d y
$$

is a version of $T_{t}^{\mu} f$ and $(t, x) \mapsto K_{t}^{\mu} f(x)$ is locally Hölder continuous on $(0, \infty) \times \mathbb{R}^{d}$. In particular, the semigroup $\left(K_{t}^{\mu}\right)_{t \geq 0}$ of kernels is strong Feller. The measure $\mu$ is a unique 
invariant probability for the semigroup $\left(K_{t}\right)_{t \geq 0}$. The proofs of these statements are given in $[1]$.

Lemma 2.7. Let $H=a^{i j} \partial_{x_{i}} \partial_{x_{j}}+b^{i} \partial_{x_{i}}$, where $a^{i j}$ and $b^{i}$ are independent of $t$ and satisfy conditions $(\mathrm{C} 1)$ and $(\mathrm{C} 2)$ with $p>d+2$ for every ball. Assume that there exists a compact function $V \in C^{2}\left(\mathbb{R}^{d}\right)$ such that $\lim _{|x| \rightarrow \infty} H V(x)=-\infty$. Let $\mu$ be a probability measure satisfying the equation $H^{*} \mu=0$ and let $\left(T_{t}^{\mu}\right)_{t \geq 0},\left(K_{t}^{\mu}\right)_{t \geq 0}$ be the corresponding semigroups introduced above. Then there exists a sequence of locally bounded measurable mappings $b_{l}: \mathbb{R}^{d} \rightarrow \mathbb{R}^{d}$ such that, letting $H_{l}:=a^{i j} \partial_{x_{i}} \partial_{x_{j}}+b_{l}^{i} \partial_{x_{i}}$, one has

(i) $b_{l} \rightarrow b$ in measure $\mu$ and $\left|b_{l}(x)\right| \leq|b(x)|$,

(ii) $\lim _{|x| \rightarrow \infty} H_{l} V(x)=-\infty$ uniformly in $l$,

(iii) there exist unique probability measures $\mu_{l}$ satisfying $H_{l}^{*} \mu_{l}=0$ such that $\mu_{l}=\varrho_{l} d x$, the functions $\varrho_{l}$ are locally uniformly Hölder continuous and converge locally uniformly to the density $\varrho$ of $\mu$, and, for the corresponding Markovian strongly continuous semigroups $(T(t, l))_{t \geq 0}$ given by strong Feller kernels $(K(t, l))_{t \geq 0}$, one has $T(t, l) f \rightarrow T_{t}^{\mu} f$ in $L^{1}(\mu)$ for all bounded measurable functions $f$. In addition, if $f \in C_{0}^{\infty}\left(\mathbb{R}^{d}\right)$, then $K(t, l) f(x) \rightarrow K_{t}^{\mu} f(x)$ for all $x \in \mathbb{R}^{d}$.

Proof. Let us fix $l \in \mathbb{N}$ and consider the closed set $Z:=\{x: \nabla V(x)=0\}$. The function $\alpha(x):=a^{i j}(x) \partial_{x_{i}} \partial_{x_{j}} V(x)$ is continuous and $H V(x)=\alpha(x)$ if $x \in Z$. Let us consider the sets $S_{m}:=\{m \leq|x|<m+1\}, m=0,1, \ldots$ One can pick $N_{l, m}$ so large that the set $\left\{x \in S_{m}:|b(x)|>N_{l, m}\right\}$ has Lebesgue measure less than $2^{-l-m}$, There exists $\delta_{m}>0$ such that if $x, x^{\prime} \in S_{m}$ and $\left|x-x^{\prime}\right|<\delta_{m}$, then $\left|\alpha(x)-\alpha\left(x^{\prime}\right)\right|<1$. We shall take $\delta_{m}=\delta_{m}(l)$ so small that the set $D_{m}:=\left\{x \in S_{m}: 0<\operatorname{dist}(x, Z) \leq \delta_{m}\right\}$ has Lebesgue measure less than $2^{-l-m}$. Now, for every $x \in D_{m}$ we set $b_{l}(x):=0$. Then we have $H_{l} V(x) \leq \sup _{y \in S_{m}} H V(y)+1$ for all $x \in D_{m}$. If $x \in S_{m} \cap Z$ and $|b(x)| \leq N_{l, m}$, we set $b_{l}(x):=b(x)$ and if $|b(x)|>N_{l, m}$, we set $b_{l}(x):=0$. Next we consider $x \in S_{m} \backslash\left(D_{m} \cup Z\right)$. Let

$$
M_{l, m}:=N_{l, m}+\left(\inf \left\{|\nabla V(y)|: y \in S_{m} \backslash\left(D_{m} \cup Z\right)\right\}\right)^{-1}\left(m+\sup _{y \in S_{m}}|\alpha(y)|\right) .
$$

Suppose that $\langle b(x), \nabla V(x)\rangle \leq-1$. If $|b(x)| \leq M_{l, m}$, then we set $b_{l}(x):=b(x)$. If $|b(x)|>$ $M_{l, m}$, then we set $b_{l}(x):=-M_{l, m} \nabla V(x) /|\nabla V(x)|$. We observe that in the latter case,

$$
\begin{aligned}
H_{l} V(x) & =\alpha(x)-M_{l, m}|\nabla V(x)| \leq \sup _{y \in S_{m}}|\alpha(y)|-M_{l, m} \inf \left\{|\nabla V(y)|: y \in S_{m} \backslash\left(D_{m} \cup Z\right)\right\} \\
& \leq-m .
\end{aligned}
$$

Finally, if $\langle b(x), \nabla V(x)\rangle>-1$, then $b_{l}(x)$ is defined to be $b(x)$ or 0 depending on whether $|b(x)| \leq N_{l, m}$ or $|b(x)|>N_{l, m}$. It follows from the above definitions that $b_{l}$ is locally bounded, $b_{l}(x)=b(x)$ outside a set of Lebesgue measure less than $2^{-l}$ and that at every point $x \in S_{m}$ one has $H_{l} V(x) \leq \sup _{y \in S_{m}} H V(y)+1$ or $H_{l} V(x) \leq-m$. Therefore, we have (i) and (ii).

As explained above, there exist unique probability measures $\mu_{l}$ such that $H_{l}^{*} \mu_{l}=0$ and that there exist unique Markovian strongly continuous semigroups $(T(t, l))_{t \geq 0}$ on $L^{1}\left(\mu_{l}\right)$ whose generators extend the operators $H_{l}$ and which are given by strongly Feller kernels $(K(t, l))_{t \geq 0}$ such that $\mu_{l}$ is invariant for $(K(t, l))_{t \geq 0}$. In addition, according to [2], the measures $\mu_{l}$ are uniformly tight and possess strictly positive locally uniformly Hölder continuous densities $\varrho_{l}$. Hence every subsequence in $\left\{\mu_{l}\right\}$ has a weak cluster point $\mu^{\prime}$. Obviously, $\mu^{\prime}$ has 
a density with respect to Lebesgue measure, hence it is readily verified that $H^{*} \mu^{\prime}=0$. Since $\mu$ is a unique probability measure satisfying this equation, we obtain $\mu^{\prime}=\mu$. Consequently, $\left\{\mu_{l}\right\}$ converges weakly to $\mu$. Together with the locally uniform Hölder continuity of the functions $\varrho_{l}$ this yields that these functions converge locally uniformly to the density $\varrho$ of $\mu$. So, in particular, $\lim _{l \rightarrow \infty}\left\|\mu_{l}-\mu\right\|=0$, where $\|\cdot\|$ denotes the variation norm.

Let $\varphi \in C_{0}^{\infty}\left(\mathbb{R}^{d}\right)$ and let $u_{l}(t, x, \varphi):=K(t, l) \varphi(x)$. Since $\left|b_{l}(x)\right| \leq|b(x)|$ and $b_{l} \rightarrow$ $b$ in measure, one can readily deduce from the results in [1] (see [1, Corollary 3.11 and Proposition 4.4]) that there is a subsequence $\left\{l_{k}\right\}$ such that the functions $u_{l_{k}}$ converge locally uniformly in $[0,+\infty) \times \mathbb{R}^{d}$ to a bounded continuous function $u(t, x, \varphi)$. Let us observe that, given any countable collection $\left\{\varphi_{n}\right\}$, one can choose such a subsequence common for all $\varphi_{n}$. In order to simply the notation we shall denote this subsequence again by $\{l\}$ (we shall see below that the assertion is indeed true for the whole sequence). We can pick the countable family $\left\{\varphi_{n}\right\}$ such that, for every bounded measurable function $f$, there exists a sequence $\left\{\varphi_{n_{k}}\right\}$ in $\left\{\varphi_{n}\right\}$ convergent to $f$ (Lebesgue) a.e. such that $\left|\varphi_{n_{k}}(x)\right| \leq \sup |f|+1$. Let $f$ be a bounded Borel measurable function. We claim that

$$
T_{t} f:=\lim _{l \rightarrow \infty} T(t, l) f
$$

exists in $L^{1}(\mu)$ and defines a Markovian strongly continuous semigroup of contractions on every $L^{p}(\mu), p \in[1, \infty)$. To prove the claim we may assume that $|f| \leq 1$. We note that for all $t \geq 0$, since the measures $\mu$ and $\mu_{l}$ are equivalent, there is no ambiguity in considering $T(t, l) f$ as an element of $L^{1}(\mu)$. Let us fix $t>0$ and $\varepsilon>0$. There exists $l_{0}$ such that $\left\|\mu-\mu_{l}\right\|<\varepsilon$ for all $l \geq l_{0}$. One can find $\varphi_{n}$ such that $\left|\varphi_{n}(x)\right| \leq 2$ and $\left\|f-\varphi_{n}\right\|_{L^{1}(\mu)}<\varepsilon$. Next we choose $l_{1}>l_{0}$ such that $\left\|T(t, l) \varphi_{n}-T(t, k) \varphi_{n}\right\|_{L^{1}(\mu)}<\varepsilon$ for all $l, k \geq l_{1}$, which is possible, since $T(t, l) \varphi_{n} \rightarrow u\left(t, \cdot, \varphi_{n}\right)$ pointwise, hence also in $L^{1}(\mu)$ by the dominated convergence theorem. We observe that for every bounded measurable function $g$, one has

$$
\begin{gathered}
\int_{\mathbb{R}^{d}}|g| d \mu \leq \int_{\mathbb{R}^{d}}|g| d \mu_{l}+\left\|\mu-\mu_{l}\right\| \sup |g(x)|, \\
\int_{\mathbb{R}^{d}}|g| d \mu_{l} \leq \int_{\mathbb{R}^{d}}|g| d \mu+\left\|\mu-\mu_{l}\right\| \sup |g(x)| .
\end{gathered}
$$

Therefore, taking into account that $T(t, l)$ is contractive on $L^{1}\left(\mu_{l}\right)$ and $|T(t, l) f| \leq 1$, $\left|T(t, l) \varphi_{n}\right| \leq 2$ for all $l$, we obtain for all $l, k \geq l_{1}$ that

$$
\begin{aligned}
& \|T(t, l) f-T(t, k) f\|_{L^{1}(\mu)} \\
& \leq\left\|T(t, l) f-T(t, l) \varphi_{n}\right\|_{L^{1}(\mu)}+\left\|T(t, l) \varphi_{n}-T(t, k) \varphi_{n}\right\|_{L^{1}(\mu)}+\left\|T(t, k) \varphi_{n}-T(t, k) f\right\|_{L^{1}(\mu)} \\
& \leq\left\|T(t, l) f-T(t, l) \varphi_{n}\right\|_{L^{1}\left(\mu_{l}\right)}+3 \varepsilon+\varepsilon+\left\|T(t, k) \varphi_{n}-T(t, k) f\right\|_{L^{1}\left(\mu_{k}\right)}+3 \varepsilon \\
& \leq\left\|f-\varphi_{n}\right\|_{L^{1}\left(\mu_{l}\right)}+\left\|f-\varphi_{n}\right\|_{L^{1}\left(\mu_{k}\right)}+7 \varepsilon \\
& \leq 2\left\|f-\varphi_{n}\right\|_{L^{1}(\mu)}+13 \varepsilon \leq 15 \varepsilon .
\end{aligned}
$$

So, $T_{t} f:=\lim _{l \rightarrow \infty} T(t, l) f$ exists in $L^{1}(\mu)$. Clearly, $T_{t}$ is Markovian. Furthermore, using (2.11) again we obtain

$$
\int_{\mathbb{R}^{d}} T_{t} f d \mu=\int_{\mathbb{R}^{d}} f d \mu
$$

since $\mu_{l}$ is invariant for $T(t, l)$. In particular, $T_{t}$ extends to a contraction on every $L^{p}(\mu)$, $p \in[1,+\infty)$. A similar reasoning shows that $\left(T_{t}\right)_{t \geq 0}$ is a semigroup on $L^{1}(\mu)$. Indeed, for all $t, s \geq 0, T_{t+s} f$ is the limit of $T(t+s, l) f=T(t, l) T(s, l) f$ in $L^{1}(\mu)$ and $T_{t} T_{s} f$ is the limit of 
$T(t, l) T_{s} f$. Let $\varepsilon>0$ and let us take $l_{0}$ such that $\left\|T(s, l) f-T_{s} f\right\|_{L^{1}(\mu)}<\varepsilon$ and $\left\|\mu-\mu_{l}\right\|<\varepsilon$ for all $l \geq l_{0}$. Then

$$
\begin{aligned}
\left\|T(t, l) T(s, l) f-T(t, l) T_{s} f\right\|_{L^{1}(\mu)} & \leq\left\|T(t, l) T(s, l) f-T(t, l) T_{s} f\right\|_{L^{1}\left(\mu_{l}\right)}+2 \varepsilon \\
& \leq\left\|T(s, l) f-T_{s} f\right\|_{L^{1}\left(\mu_{l}\right)}+2 \varepsilon \\
& \leq\left\|T(s, l) f-T_{s} f\right\|_{L^{1}(\mu)}+4 \varepsilon<5 \varepsilon
\end{aligned}
$$

Since $t \mapsto T_{t} \varphi_{n}$ is continuous from $[0,+\infty)$ to $L^{p}(\mu)$ for all $n,\left\{\varphi_{n}\right\}$ is dense in $L^{p}(\mu)$ and each $T_{t}$ is a contraction on $L^{p}(\mu)$, we obtain the strong continuity of $\left(T_{t}\right)_{t \geq 0}$. The claim is proved.

To complete the proof of this lemma it remains to show that

$$
T_{t}^{\mu} f=T_{t} f \quad \forall t>0, f \in L^{1}(\mu) .
$$

Indeed, (2.12) implies that $T_{t}$ is independent of the subsequence chosen in its definition, so $T(t, l) f \rightarrow T_{t}^{\mu} f$ in $L^{1}(\mu)$ for all bounded measurable functions $f$. Furthermore, for all $\varphi \in C_{0}^{\infty}\left(\mathbb{R}^{d}\right)$ and $t>0$, by continuity $K_{t}^{\mu} \varphi(x)=u(t, x, \varphi)$ for all $x \in \mathbb{R}^{d}$, so the last part of assertion (iii) follows from (2.12). Let $(\widehat{H}, D(\widehat{H}))$ be the generator of $\left(T_{t}\right)_{t \geq 0}$ on $L^{1}(\mu)$. By virtue of the uniqueness result mentioned before this lemma, it suffices to show that for any $\varphi \in C_{0}^{\infty}\left(\mathbb{R}^{d}\right)$, one has

$$
\varphi \in D(\widehat{H}) \text { and } \widehat{H} \varphi=H \varphi,
$$

which in turn follows from the identity

$$
\int_{\mathbb{R}^{d}} T_{t} \varphi g d \mu=\int_{\mathbb{R}^{d}} \varphi g d \mu+\int_{0}^{t} \int_{\mathbb{R}^{d}} T_{s}(H \varphi) g d \mu d s, \quad \forall t>0, g \in C_{0}^{\infty}\left(\mathbb{R}^{d}\right) .
$$

To prove (2.13) we need some preparations. Let $l \in \mathbb{N}$. Consider the operator $\left(H_{l}^{\prime}, C_{0}^{\infty}\left(\mathbb{R}^{d}\right)\right)$ on $L^{2}\left(\mu_{l}\right)$ defined by

$$
H_{l}^{\prime} \varphi:=a^{i j} \partial_{x_{i}} \partial_{x_{j}} \varphi+\left(2 \partial_{x_{i}} a^{i j}+2 a^{i j} \partial_{x_{i}} \varrho_{l} / \varrho_{l}-b_{l}^{i}\right) \partial_{x_{j}} \varphi .
$$

Then $\left(H_{l}^{\prime}\right)^{*} \mu_{l}=0$ and by [14] the closure of this operator generates a Markovian strongly continuous semigroup of contractions $T^{\prime}(t, l)$ on $L^{1}\left(\mu_{l}\right)$ such that for all bounded measurable $f$ and all $g$ one has

$$
\int_{\mathbb{R}^{d}} T(t, l) f g d \mu_{l}=\int_{\mathbb{R}^{d}} f T^{\prime}(t, l) g d \mu_{l}
$$

By [3, Remark 2.14] the functions $\partial_{x_{i}} \varrho_{l} / \varrho_{l}$ have uniformly bounded local $L^{p}$-norms. Therefore, by the same arguments as above, $T_{t}^{\prime} f:=\lim _{l \rightarrow \infty} T^{\prime}(t, l) f$ exists in $L^{1}(\mu)$ for all bounded measurable functions $f$ (for some subsequence of $\left\{l_{k}\right\}$ again denoted by $\{l\}$ ). By (2.11) we obtain for all bounded measurable $f$ and $g$

$$
\int_{\mathbb{R}^{d}} T_{t} f g d \mu=\int_{\mathbb{R}^{d}} f T_{t}^{\prime} g d \mu .
$$

Clearly, (2.14) extends to all $g \in L^{1}(\mu)$. Now we can prove (2.13). Let $\varphi, g \in C_{0}^{\infty}\left(\mathbb{R}^{d}\right)$. Then

$$
\begin{aligned}
\int_{\mathbb{R}^{d}} T_{t} \varphi g d \mu & =\lim _{l \rightarrow \infty} \int_{\mathbb{R}^{d}} T(t, l) \varphi g d \mu=\lim _{l \rightarrow \infty} \int_{\mathbb{R}^{d}} T(t, l) \varphi g d \mu_{l} \\
& =\lim _{l \rightarrow \infty}\left(\int_{\mathbb{R}^{d}} \varphi g d \mu_{l}+\int_{0}^{t} T(t, l) \int_{\mathbb{R}^{d}} T(s, l) H_{l} \varphi g d \mu_{l} d s\right) \\
& =\int_{\mathbb{R}^{d}} \varphi g d \mu+\lim _{l \rightarrow \infty} \int_{0}^{t} T(t, l) \int_{\mathbb{R}^{d}} T(s, l) H \varphi g d \mu_{l} d s,
\end{aligned}
$$


since $\lim _{l \rightarrow \infty}\left\|H_{l} \varphi-H_{l} \varphi\right\|_{L^{2}\left(\mu_{l}\right)}$ due to the uniform boundedness of $L^{2}(U)$-norms of $\varrho_{l}$ on every ball $U$. It remains to note that on the support of $\varphi$ the densities $\varrho_{l}$ converge uniformly to $\varrho$ (which is strictly positive continuous), which yields

$$
\begin{aligned}
\lim _{l \rightarrow \infty} \int_{\mathbb{R}^{d}} T(s, l) H \varphi g d \mu_{l} & =\lim _{l \rightarrow \infty} \int_{\mathbb{R}^{d}} H \varphi T(s, l)^{\prime} g d \mu_{l} \\
& =\int_{\mathbb{R}^{d}} H \varphi T_{s}^{\prime} g d \mu=\int_{\mathbb{R}^{d}} T_{s} H \varphi g d \mu .
\end{aligned}
$$

In addition, these integrals are uniformly bounded in $s$. Hence we arrive at (2.13).

Remark 2.8. We observe that the existence of a Lyapunov function $V$ was only used in order to obtain the following three properties: (a) the very existence of the measures $\mu^{l}$ and $\mu$ satisfying $H_{l}^{*} \mu_{l}=0, H^{*} \mu=0$ and generating the semigroups $(T(t, l))_{t \geq 0}$ and $\left(T_{t}^{\mu}\right)_{t \geq 0}$, for which they are invariant; (b) the convergence of $\mu_{l}$ to $\mu$ in the variation norm; (c) the uniqueness of a probability measure satisfying $H^{*} \mu=0$ and the uniqueness of the associated Markovian semigroup. Therefore, the same assertion is true if we require (a), (b) and (c) in place of the existence of $V$, assuming that $b_{l} \rightarrow b$ in measure $\mu$ and $\left|b_{l}(x)\right| \leq|b(x)|+1$ (and keeping our usual local assumptions on $a^{i j}$ and $b^{i}$ ).

Lemma 2.9. Let $p>d+2$. Let us assume that there exist points $0=\tau_{0}<\tau_{1}<\cdots<\tau_{n}<1$ such that $A(t, x)=A\left(\tau_{k}, x\right)$ and $b(t, x)=b\left(\tau_{k}, x\right)$ if $t \in\left[\tau_{k}, \tau_{k+1}\right)$. Suppose also that, for every fixed ball $U \subset \mathbb{R}^{d}$, one has $\inf _{x \in U} \operatorname{det} A\left(\tau_{k}, x\right)>0, a^{i j}\left(\tau_{k}, \cdot\right) \in H^{p, 1}(U), b^{i}\left(\tau_{k}, \cdot\right) \in L^{p}(U)$ for each $k$. Finally, assume that there exist nonnegative compact functions $V_{l} \in C^{2}\left(\mathbb{R}^{d}\right)$ such that one has

$$
\lim _{|x| \rightarrow \infty}\left[a^{i j}\left(\tau_{k}, x\right) \partial_{x_{i}} \partial_{x_{j}} V_{k}(x)+b^{i}\left(\tau_{k}, x\right) \partial_{x_{i}} V_{k}(x)\right]=-\infty
$$

Then, for every probability measure $\nu$, there exists a family $\mu=\left(\mu_{t}\right)_{t \in[0,1)}$ of probability measures on $\mathbb{R}^{d}$ satisfying (1.3) and (1.5) such that $t \mapsto \int_{\mathbb{R}^{d}} \zeta d \mu_{t}$ is continuous on $[0,1)$ for all $\zeta \in C_{0}^{\infty}\left(\mathbb{R}^{d}\right)$.

Proof. Suppose first that the mappings $\left|b\left(\tau_{k}, \cdot\right)\right|$ are locally bounded. As explained in the proof of Lemma 2.7, for every $k$ there exist a probability measure $\mu^{k}$ on $\mathbb{R}^{d}$ and a strongly continuous Markovian semigroup $(T(t, k))_{t \geq 0}$ on $L^{1}\left(\mu^{k}\right)$ such that its generator extends the operator

$$
H_{k}:=a^{i j}\left(\tau_{k}, x\right) \partial_{x_{i}} \partial_{x_{j}}+b^{i}\left(\tau_{k}, x\right) \partial_{x_{i}}
$$

and $\mu^{k}$ is invariant for $T(t, k)$. Let $K(t, k)$ denote the corresponding Markovian kernels. Let us set

$$
\mu_{t}:=K\left(t-\tau_{k}, k\right)^{*} K\left(\tau_{k}, k-1\right)^{*} \cdots K\left(\tau_{1}, 0\right)^{*} \nu \text { if } t \in\left[\tau_{k}, \tau_{k+1}\right) .
$$

We shall verify that $\left(\mu_{t}\right)_{t \in[0,1)}$ satisfies (1.3) and (1.5). Indeed, (1.5) is true according to [1, Proposition 4.4]. In order to verify (1.3), it suffices to consider $u$ of the form $\varphi(t) u(x)$ with $\varphi \in C_{0}^{\infty}(0,1)$ and $u \in C_{0}^{\infty}\left(\mathbb{R}^{d}\right)$. For each $k$ we have

$$
\begin{aligned}
& \int_{\tau_{k}}^{\tau_{k+1}} \int_{\mathbb{R}^{d}}\left[\varphi^{\prime}(t) u(x)+\varphi(t) H_{k} u(x)\right] K\left(t-\tau_{k}, k\right)^{*} \mu_{\tau_{k}}(d x) d t \\
& =\varphi\left(\tau_{k+1}\right) \int_{\mathbb{R}^{d}} u(x) \mu_{\tau_{k+1}}(d x)-\varphi\left(\tau_{k}\right) \int_{\mathbb{R}^{d}} u(x) \mu_{\tau_{k}}(d x) .
\end{aligned}
$$

Here we use that $\frac{d}{d t} T\left(t-\tau_{k}, k\right) u=T\left(t-\tau_{k}, k\right) H_{k} u$, where $\frac{d}{d t}$ is taken in $L^{1}\left(\mu_{\tau_{k}}\right)$. Indeed, this holds by the following reasoning: we know that this is true if $\frac{d}{d t}$ is taken in $L^{1}\left(\mu^{k}\right)$, 
hence it can be taken in $\mu^{k}$-measure (which is equivalent to Lebesgue measure). Since $\mu_{\tau_{k}}$ is absolutely continuous (which is proved in [1]) and since $H_{k} u$ is a bounded function (this is the only place where we use the local boundedness of $\left.b\left(\tau_{k}, \cdot\right)\right)$, it follows by the dominated convergence theorem that $\frac{d}{d t}$ can be taken in $L^{1}\left(\mu_{\tau_{k}}\right)$. Taking into account the equality $\varphi(0)=\varphi(1)=0$, we arrive at $(1.3)$.

Let us consider the general case. According to Lemma 2.7, for each $k=0, \ldots, n$, there exists a sequence of locally bounded mappings $b_{l}\left(\tau_{k}, \cdot\right)$ such that $\left|b_{l}\left(\tau_{k}, x\right)\right| \leq\left|b\left(\tau_{k}, x\right)\right|+1$ and $\lim _{|x| \rightarrow \infty} H_{k, l} V(x)=-\infty$ uniformly in $l$, where

$$
H_{k, l} \varphi(x):=a^{i j}\left(\tau_{k}, x\right) \partial_{x_{i}} \partial_{x_{j}} \varphi(x)+b_{l}^{i}\left(\tau_{k}, x\right) \partial_{x_{i}} \varphi(x)
$$

Let

$$
\mathcal{H}_{l}(t, x):=a^{i j}\left(\tau_{k}, x\right) \partial_{x_{i}} \partial_{x_{j}} \varphi(x)+b_{l}^{i}\left(\tau_{k}, x\right) \partial_{x_{i}} \varphi(x) \quad \text { if } \quad t \in\left[\tau_{k}, \tau_{k+1}\right), k=0, \ldots, n .
$$

As shown above, for each $b_{l}$ there exists a solution $\left(\mu_{t}^{l}\right)_{t \in[0,1)}$ of the problem (1.3) and (1.5) corresponding to $\mathcal{H}_{l}$. The semigroups corresponding to the drifts $b_{l}\left(\tau_{k}, x\right)$ (used above to construct solutions for $\left.\mathcal{H}_{l}\right)$ will be denoted by $T(t, k, l)$ and $K(t, k, l)$, respectively. As shown in [1], the measures $\mu_{t}^{l}$ have densities $\varrho(l, t)$ such that, for every interval $[c, d] \subset(0,1)$ and every closed ball $B \subset \mathbb{R}^{d}$, the functions $\Phi_{l}:(t, x) \mapsto \varrho(l, t)(x)$ are Hölder continuous on $[c, d] \times B$ uniformly in $l$. Therefore, passing to a subsequence, we may assume that the sequence $\left\{\Phi_{l}\right\}$ converges locally uniformly on $(0,1) \times \mathbb{R}^{d}$ to a continuous function $\Phi$. It is readily seen that the family of measures $\mu_{t}:=\Phi(t, x) d x$ satisfies (1.3). We observe that the sequence of measures $\mu_{t}^{l}$ is uniformly tight for each $t \in[0,1)$. Indeed, by Lemma 2.2, the family of measures $\mu_{t}^{l}, t \in\left[0, \tau_{1}\right], l \in \mathbb{N}$, is uniformly tight. It follows by Corollary 2.4 that the family of measures $\mu_{t}^{l}, t \in\left[\tau_{1}, \tau_{2}\right], l \in \mathbb{N}$, is uniformly tight as well. Repeatedly applying Corollary 2.4 we obtain the uniform tightness of the measures $\mu_{t}^{l}, t \in[0,1), l \in \mathbb{N}$. Together with the convergence of densities this yields that the measures $\mu_{t}$ are probabilities. It remains to verify (1.5). Let $\zeta \in C_{0}^{\infty}\left(\mathbb{R}^{d}\right)$. It suffices to show that the functions

$$
t \mapsto g_{l}(t):=\int_{\mathbb{R}^{d}} \zeta d \mu_{t}^{l}
$$

are uniformly continuous on $[0,1)$ and are equal to $\int \zeta d \nu$ at $t=0$. We have for $t \in\left[0, \tau_{1}\right]$ and $u(t, \cdot, l):=K(t, 0, l) \zeta$ that

$$
g_{l}(t)=\int_{\mathbb{R}^{d}} u(t, x, l) \nu(d x)
$$

So, $g_{l}(0)=\int_{\mathbb{R}^{d}} \zeta d \nu$ for all $l$.

We claim that $u(t, \cdot, l), t \in\left[0, \tau_{1}\right]$, satisfies (2.9). Suppose the claim is proved. Then by Lemma 2.6 and our assumptions on $b_{l}$, the functions $s \mapsto u(t, x, l)$ are Hölder continuous on $\left[0, \tau_{1}\right]$ uniformly in $l, x \in B, t \in\left[0, \tau_{1}\right]$, whence it follows that the functions $g_{l}$ are equicontinuous. Indeed, given $\varepsilon>0$, one can find a ball $B$ such that $\nu(B)>1-\varepsilon / 4$. There exists $\delta=\delta(\varepsilon)>0$ such that $\left|u(t, x, l)-u\left(t^{\prime}, x, l\right)\right|<\varepsilon / 4$ for all $x \in B$ and all $l$ if $\left|t-t^{\prime}\right|<\delta$. Hence

$$
\left|g_{l}(t)-g_{l}\left(t^{\prime}\right)\right| \leq \frac{\varepsilon}{2}+2 \nu\left(\mathbb{R}^{d} \backslash B\right)<\varepsilon
$$

for all $l$ if $\left|t-t^{\prime}\right|<\delta$. In particular, we obtain that $\lim _{l \rightarrow \infty} g_{l}(t)=\int \zeta d \nu$ uniformly in $l$, which yields (1.5). It remains to prove the claim. To this end we first note that by exactly the same arguments as used above we see that

$$
\frac{d}{d t} u(t, \cdot, l)=H_{0, l} u(t, \cdot, l)=T(t, l) H_{0, l} \zeta
$$


with $\frac{d}{d t}$ taken in $L_{l o c}^{1}(d x)$ (instead of $\left.L^{1}\left(\mu^{0}\right)\right)$. Furthermore, $|u(t, \cdot, l)| \leq\|\zeta\|_{\infty}$ and $u(t, \cdot, l) \in$ $H_{l o c}^{p, 1}\left(\mathbb{R}^{d}\right)$ by $\left[1\right.$, Corollary 2.13]. Let $\varphi \in C_{0}^{\infty}\left(-\infty, \tau_{1}\right) \times \mathbb{R}^{d}$. Then we have for all $u(l):=$ $u(\cdot, \cdot, l)$ integrating by parts

$$
\begin{aligned}
& \int_{0}^{1} \int_{\mathbb{R}^{d}}\left[-u(l) \partial_{t} \varphi+a^{i j} \partial_{x_{i}} u(l) \partial_{x_{j}} \varphi+\varphi \partial_{x_{i}} a^{i j} \partial_{x_{j}} u(l)-\varphi b_{l}^{i} \partial_{x_{i}} u(l)\right] d x d t \\
& =-\int_{0}^{1} \int_{\mathbb{R}^{d}} \frac{d}{d t}(u(l) \varphi) d x d t-\int_{0}^{1} \int_{\mathbb{R}^{d}} H_{0, l} u(l) \varphi d x d t+\int_{0}^{1} \int_{\mathbb{R}^{d}} \frac{d}{d t} u(l) \varphi d x d t \\
& =\int_{\mathbb{R}^{d}} \zeta(x) \varphi(0, x) d x .
\end{aligned}
$$

This completes the proof.

\section{MAin RESUlts}

Theorem 3.1. Let $p>d+2$ and let $A$ and $b$ satisfy $(\mathrm{C} 1)$ and $(\mathrm{C} 2)$ for every ball. Assume that for a.e. $t \in(0,1)$, there exist a nonnegative compact function $V_{t} \in C^{2}\left(\mathbb{R}^{d}\right)$ such that one has

$$
\lim _{|x| \rightarrow \infty}\left[a^{i j}(t, x) \partial_{x_{i}} \partial_{x_{j}} V_{t}(x)+b^{i}(t, x) \partial_{x_{i}} V_{t}(x)\right]=-\infty .
$$

Finally, assume that there exists a nonnegative compact function $\Psi \in C^{2}\left(\mathbb{R}^{d}\right)$ and a constant $C \geq 0$ such that

$$
L \Psi \leq C \quad \text { a.e. in }(0,1) \times \mathbb{R}^{d} .
$$

Then, for every probability measure $\nu$, there exists a family $\mu=\left(\mu_{t}\right)_{t \in[0,1)}$ of probability measures on $\mathbb{R}^{d}$ satisfying (1.3) and (1.5) such that $t \mapsto \int_{\mathbb{R}^{d}} \zeta d \mu_{t}$ is continuous on $[0,1)$ for every $\zeta \in C_{0}^{\infty}\left(\mathbb{R}^{d}\right)$.

Proof. We may assume that (3.1) holds for all $t \in(0,1)$, since for those $t$ which do not satisfy this condition, we may redefine $A$ and $b$ by setting $A(t, x)=A\left(t_{0}, x\right), b(t, x)=b\left(t_{0}, x\right)$, where $t_{0}$ is any fixed point for which that condition is satisfied. Clearly, this does not affect other hypotheses and (1.3) is not sensitive to such redefinitions of the coefficients.

Our strategy of proof is as follows. We have already proved the result in the case when $A(t, x)$ and $b(t, x)$ are piece-wise constant in $t$. Now we consider a sequence of solutions $\mu^{k}=$ $\left(\mu_{t}^{k}\right)_{t \in[0,1)}$ corresponding to $A_{k}(t, x)$ and $b_{k}(t, x)$ which coincide with $A\left(t_{k, j}, x\right)$ and $b\left(t_{k, j}, x\right)$ if $t \in\left[t_{k, j}, t_{k, j+1}\right)$, where $0=t_{k, 0}<t_{k, 1}<\ldots<t_{k, N_{k}}=1$ is a suitably chosen partition of $(0,1]$ into $N_{k}$ intervals. We shall verify that the measures $\mu_{t}^{k}, t \in[0,1), k \in \mathbb{N}$, are uniformly tight on $\mathbb{R}^{d}$. Finally, the desired solution $\mu$ will be constructed as a weak cluster point of $\left\{\mu^{k}\right\}$. In order to show that $\mu$ satisfies our equation, certain uniform a priori estimates for $\left\{\mu^{k}\right\}$ will be established. First of all, let us choose a sequence of decreasing partitions of $[0,1)$ as follows (in the case when $A$ and $b$ are continuous in $t$, one can take $t_{k, j}=j 2^{-k}, N_{k}=2^{k}$, which does not work in general as we shall see). We recall a well-known result of Lebesgue according to which, for any integrable function $f$ on $[0,1]$, one can find a decreasing sequence of partitions $\left[0, t_{k, 1}\right),\left[t_{k, 1}, t_{k, 2}\right), \ldots,\left[t_{k, N_{k}}, 1\right)$ (i.e., every partition contains all partition points of the previous one) such that the Riemann sums $\sum_{j=1}^{N_{k}} f\left(t_{k, j-1}\right)\left(t_{k, j}-t_{k, j-1}\right)$, where $t_{k, 0}=0$, converge to the integral of $f$. We shall call such a sequence of partitions a Riemannian sequence of partitions. Moreover, given a countable family of integrable functions $f_{n}$, one can choose common points $t_{k, j}$ for all $f_{n}$. The simplest way to produce such partitions is 
to employ the fact established by Jessen [9] that, for any function $f$ that has period 1 and is integrable on $[0,1]$, the Riemann sums $R_{k}(f, s):=2^{-k} \sum_{j=1}^{2^{k}} f\left(s+j 2^{-k}\right)$ converge to the integral of $f$ over $[0,1]$ for almost all $s$. We shall apply Lebesgue's result to the following countable collection of functions. Let $\left\{\zeta_{n}\right\} \subset C_{0}^{\infty}(0,1)$ and $\left\{\varphi_{m}\right\} \subset C_{0}^{\infty}\left(\mathbb{R}^{d}\right)$ be two sequences of functions with the following property: for every function $u$ that is continuous and has compact support in $(0,1) \times \mathbb{R}^{d}$, there exists a sequence $h_{j}$ of finite linear combinations of the functions $\zeta_{n} \varphi_{m}$ with rational coefficients such that the functions $h_{j}$ vanish outside some compact set containing the support of $u$ and converge to $u$ uniformly. Let us consider functions $\zeta_{n}(t) \alpha_{i, j, m}(t)$ and $\zeta_{n}(t) \beta_{i, j, m}(t)$, where

$$
\alpha_{i, j, m}(t)=\int_{\mathbb{R}^{d}} \varphi_{m}(x) a^{i j}(t, x) d x, \quad \beta_{i, m}(t)=\int_{\mathbb{R}^{d}} \varphi_{m}(x) b^{i}(t, x) d x .
$$

Let us choose for the obtained countable set of functions $\zeta_{n} \alpha_{i, j, m}, \zeta_{n} \beta_{i, j, m}$ a common Riemannian sequence of decreasing partitions formed by the points $t_{k, j}, j=0, \ldots, N_{k}$. Let us set $A_{k}=\left(a_{k}^{i k}\right), b_{k}=\left(b_{k}^{i}\right)$, where

$$
a_{k}^{i j}(t, x)=a^{i j}\left(t_{k, l-1}, x\right), b_{k}^{i}(t, x)=b^{i}\left(t_{k, l-1}, x\right) \quad \text { if } t \in\left[t_{k, l-1}, t_{k, l}\right) .
$$

We observe that the following is true. Let $u \in C_{0}^{\infty}\left((0,1) \times \mathbb{R}^{d}\right)$, let $J$ be a closed interval in $(0,1)$, let $K$ be a closed ball in $\mathbb{R}^{d}$ such that $\operatorname{supp} u \subset J \times K$, and let $\left\{\varrho_{n}\right\}$ be a sequence of continuous functions that converges to a function $\varrho$ uniformly on $J \times K$. Then, for all $i, j$ one has

$$
\begin{aligned}
& \lim _{k \rightarrow \infty} \int_{0}^{1} \int_{\mathbb{R}^{d}} a_{k}^{i j}(t, x) u(t, x) \varrho_{k}(t, x) d x d t=\int_{0}^{1} \int_{\mathbb{R}^{d}} a^{i j}(t, x) u(t, x) \varrho(t, x) d x d t \\
& \lim _{k \rightarrow \infty} \int_{0}^{1} \int_{\mathbb{R}^{d}} b_{k}^{i}(t, x) u(t, x) \varrho_{k}(t, x) d x d t=\int_{0}^{1} \int_{\mathbb{R}^{d}} b^{i}(t, x) u(t, x) \varrho(t, x) d x d t .
\end{aligned}
$$

Let us verify (3.3). Considering the functions $u \varrho_{n}$ and $u \varrho$ we arrive at the case where $u=1$ and the functions $\varrho_{n}$ vanish outside $J \times K$. It follows from their uniform convergence that it suffices to show that

$$
\lim _{k \rightarrow \infty} \int_{0}^{1} \int_{\mathbb{R}^{d}} a_{k}^{i j}(t, x) \varrho(t, x) d x d t=\int_{0}^{1} \int_{\mathbb{R}^{d}} a^{i j}(t, x) \varrho(t, x) d x d t .
$$

In turn, due to our choice of $\left\{\zeta_{n}\right\}$ and $\left\{\varphi_{m}\right\}$, it is enough to consider $\varrho(t, x)=\zeta_{n}(t) \varphi_{m}(x)$, i.e., to show that

$$
\lim _{k \rightarrow \infty} \sum_{l=1}^{N_{k}} \alpha_{i, j, m}\left(t_{k, l-1}\right) \int_{t_{k, l-1}}^{t_{k, l}} \zeta_{n}(t) d t=\int_{0}^{1} \zeta_{n}(t) \alpha_{i, j, m}(t) d t .
$$

According to our construction, we have

$$
\lim _{k \rightarrow \infty} \sum_{l=1}^{N_{k}} \zeta_{n}\left(t_{k, l}\right) \alpha_{i, j, m}\left(t_{k, l-1}\right)\left(t_{k, l}-t_{k, l-1}\right)=\int_{0}^{1} \zeta_{n}(t) \alpha_{i, j, m}(t) d t
$$


Now it suffices to note that, letting $\delta_{k}=\max _{l \leq N_{k}}\left|t_{k, l}-t_{k, l-1}\right|$, one has

$$
\begin{aligned}
& \left|\sum_{l=1}^{N_{k}} \alpha_{i, j, m}\left(t_{k, l-1}\right) \int_{t_{k, l-1}}^{t_{k, l}} \zeta_{n}(t) d t-\sum_{l=1}^{N_{k}} \zeta_{n}\left(t_{k, l-1}\right) \alpha_{i, j, m}\left(t_{k, l}\right)\left(t_{k, l}-t_{k, l-1}\right)\right| \\
& \leq \sup _{s \in(0,1)}\left|\alpha_{i, j, m}(s)\right| \sup _{t, s:|t-s| \leq \delta_{k}}\left|\zeta_{n}(t)-\zeta_{n}(s)\right|,
\end{aligned}
$$

which tends to 0 as $k \rightarrow \infty$ by the uniform continuity of $\zeta_{n}$. The same reasoning proves (3.4).

We now turn to the main statement. According to Lemma 2.9, for the $k$-th partition, we have a solution $\mu^{k}=\left(\mu_{t}^{k}\right)_{t \in[0,1)}$ of our problem corresponding to the operator $H_{k}$ given by $a_{k}^{i j}(t, x)=a\left(t_{k, j}, x\right), b_{k}^{i}(t, x)=b\left(t_{k, j}, x\right)$ if $t \in\left[t_{k, j}, t_{k, j+1}\right)$. We have $\mu_{t}^{k}=\varrho_{k}(t, x) d x$, where the functions $\varrho_{k}(t, x)$ are jointly continuous on $(0,1) \times \mathbb{R}^{d}$. According to Corollary 2.4, there is a nonnegative function $\Psi \in C^{2}\left(\mathbb{R}^{d}\right)$ such that $\Psi(x) \rightarrow+\infty$ as $|x| \rightarrow+\infty, L \Psi \leq C$ and $\Psi \in L^{1}(\nu)$. By Lemma 2.2, the family of measures $\mu_{t}^{k}, t \in[0,1), k \in \mathbb{N}$, is uniformly tight. By the same reasoning as in Lemma 2.9, passing to a subsequence, we may assume that the functions $\varrho_{k}(t, x)$ converge uniformly on compact subsets in $(0,1) \times \mathbb{R}^{d}$ to a function $\varrho(t, x)$. Therefore, we obtain probability measures $\mu_{t}:=\varrho(t, x) d x$, which satisfy (1.3).

The last step is to show that $\mu:=\left(\mu_{t}\right)_{t \in[0,1)}$ satisfies (1.5). It suffices to show that, for every $\zeta \in C_{0}^{\infty}\left(\mathbb{R}^{d}\right)$, the functions

$$
g_{k}(t)=\int_{\mathbb{R}^{d}} \zeta(x) \mu_{t}^{k}(d x)
$$

are equicontinuous on $[0,1 / 2]$. However, this is clear from the analogous step in the proof of Lemma 2.9, since every $g_{k}$ is the limit of the functions $g_{k, l}$ corresponding to the approximations constructed in the lemma cited (see (2.16)), and those functions are equicontinuous also with respect to $k$, which follows from the proof of the equicontinuity of the functions in (2.16).

Remark 3.2. (i) It is clear from the proof of the above theorem that in the case when the functions $b^{i}$ are bounded on bounded subsets of $(0,1) \times \mathbb{R}^{d}$, the nondegeneracy condition on $A$ can be slightly relaxed as follows: it suffices to have

$$
\inf _{(t, x) \in\left[\tau_{1}, \tau_{2}\right] \times K} \operatorname{det} A(t, x)>0
$$

for every $\left[\tau_{1}, \tau_{2}\right] \subset(0,1)$ and every compact set $K \subset \mathbb{R}^{d}$.

(ii) It follows from Remark 2.5 and the above proof that condition (3.2) can be relaxed as follows: there exists a compact set $K \subset \mathbb{R}^{d}$ such that $L \Psi(t, x) \leq C$ a.e. in $(0,1) \times\left(\mathbb{R}^{d} \backslash K\right)$.

(iii) It is also clear that the solution constructed above has the following property: for a.e. $t$, the measure $\mu_{t}$ has a density from the Sobolev class $H_{l o c}^{p, 1}\left(\mathbb{R}^{d}\right)$. As shown in [1], this is true for any solution of (1.3) under our local assumptions on $A$ and $b$. Hence, under these assumptions, equation (1.3) can be written in the classical weak form after integrating by parts in the term with $\partial_{x_{i}} \partial_{x_{j}} u$. Below we consider more general equations whose solutions do not have such a property.

Corollary 3.3. Suppose that $\operatorname{det} A(t, x)$ is uniformly bounded on $[0,1) \times \mathbb{R}^{d}$ and, for every compact set $K \subset \mathbb{R}^{d}$ and every $\left[\tau_{1}, \tau_{2}\right] \subset(0,1)$, one has

$$
\inf _{(t, x) \in\left[\tau_{1}, \tau_{2}\right] \times K} \operatorname{det} A(t, x)>0, \sup _{(t, x) \in[0,1) \times K}|b(t, x)|<\infty .
$$


Assume also that

$$
\lim _{|x| \rightarrow \infty}\langle b(t, x), x\rangle=-\infty \quad \text { for a.e. } t \in(0,1) \text { and } \sup _{(t, x) \in[0,1) \times \mathbb{R}^{d}}\langle b(t, x), x\rangle=M<\infty .
$$

Then, for every probability measure $\nu$ on $\mathbb{R}^{d}$, there exists a family $\left(\mu_{t}\right)_{t \in[0,1)}$ of probability measures on $\mathbb{R}^{d}$ satisfying (1.3) and (1.5) such that $t \mapsto \int_{\mathbb{R}^{d}} \zeta d \mu_{t}$ is continuous on $[0,1)$ for every $\zeta \in C_{0}^{\infty}\left(\mathbb{R}^{d}\right)$.

If, in addition, the functions $b^{i}$ and $a^{i j}$ are continuous in $x$ for a.e. fixed $t$, then the same is true without the assumption that $\operatorname{det} A$ is strictly positive.

Proof. As in the theorem, we may assume that the first relation in (3.5) holds for each $t$. We shall find a sequence of smooth mappings $A_{j}(t, x)$ such that

$$
\sup _{j} \sup _{(t, x) \in[0,1) \times \mathbb{R}^{d}}\left\|A_{j}(t, x)\right\| \leq S+1<\infty
$$

and, for every compact set $K \in \mathbb{R}^{d}$ and every $\left[\tau_{1}, \tau_{2}\right] \subset(0,1)$, one has

$$
\inf _{j} \inf _{(t, x) \in\left[\tau_{1}, \tau_{2}\right] \times K} \operatorname{det} A_{j}(t, x)>0 .
$$

Then we shall verify that the sequence of the corresponding solutions $\left(\mu_{t}^{j}\right)_{t \in[0,1)}$ has a subsequence which is weakly convergent for each $t$ and that its weak limit is the desired solution. The approximations $A_{j}$ can be given by $A * j^{d} \theta(j x)+j^{-1} \mathrm{I}$, where $\theta$ is a smooth probability density on $\mathbb{R}^{d}$ with support in the unit ball $U$. Then (3.6) is clear and, for each fixed $j$, the functions $\partial_{x_{m}} a_{j}^{i k}$ are uniformly bounded on bounded subsets in $(0,1) \times \mathbb{R}^{d}$ and $\operatorname{det} A_{j} \geq j^{-d}$. In addition, given $\left[\tau_{1}, \tau_{2}\right] \subset(0,1)$ and $k>0$, for every unit vector $e$ and all $(t, x) \in\left[\tau_{1}, \tau_{2}\right] \times k U$, one has

$$
\begin{aligned}
\left\langle A_{j}(t, x) e, e\right\rangle & =j^{-1}+\int_{U}\langle A(t, x-y / j) e, e\rangle \theta(y) d y \geq \inf _{(t, z) \in\left[\tau_{1}, \tau_{2}\right] \times(k+1) U}\langle A(t, z) e, e\rangle \\
& \geq \inf _{(t, z) \in\left[\tau_{1}, \tau_{2}\right] \times(k+1) U}(\operatorname{det} A(t, z))^{1 / d}>0 .
\end{aligned}
$$

Let us denote by $L_{j}$ the parabolic operator obtained from $L$ by replacing $A$ with $A_{j}$. Let us set $V_{t}(x)=\langle x, x\rangle$. Then, since $\sup _{j, t, x}\left\|A_{j}(t, x)\right\| \leq S+1$, we obtain for every fixed $t$ that $\lim _{|x| \rightarrow \infty} L_{j} V_{t}=-\infty$. Let us fix a probability measure $\nu$ on $\mathbb{R}^{d}$. Letting $\Psi_{0}(x)=\langle x, x\rangle$, one has $L \Psi_{0} \leq 2 d S+2 M$. Therefore, by Corollary 2.4 , we can replace $\Psi_{0}$ with a nonnegative compact function $\Psi \in C^{2}\left(\mathbb{R}^{d}\right)$ such that $L \Psi \leq 2 d S+2 M$ and $\Psi \in L^{1}(\nu)$. In addition, $\Psi$ constructed in that corollary has the form $\Psi=\theta\left(\Psi_{0}\right)$ with $0 \leq \theta^{\prime} \leq 1$ and $\theta^{\prime \prime} \leq 0$. Hence

$$
\begin{aligned}
L_{j} \Psi(t, x) & =2 \theta^{\prime}\left(|x|^{2}\right) \operatorname{trace} A_{j}(t, x)+4 \theta^{\prime \prime}\left(|x|^{2}\right)\left\langle A_{j}(t, x) x, x\right\rangle \\
& +2 \theta^{\prime}\left(|x|^{2}\right)\langle b(t, x), x\rangle \leq 2 d S+2 M .
\end{aligned}
$$

According to the above theorem, for each $j$, there exists a family of probability measures $\left(\mu_{t}^{j}\right)_{t \in[0,1)}$ on $\mathbb{R}^{d}$ satisfying (1.3) and (1.5) for the operator $L_{j}$. In addition, $\mu_{t}^{j}=f_{j}(t, x) d x$ for $t>0$. It follows by Lemma 2.2 that, for every $t \in[0,1)$, the sequence of measures $\mu_{t}^{j}$ is uniformly tight. Let $\varphi \in C_{0}^{\infty}\left(\mathbb{R}^{d}\right)$ be fixed. Since the functions $\left|a_{j}^{i k}\right|,\left|\partial_{x_{i}} \partial_{x_{k}} \varphi\right|$, and $|\langle\nabla \varphi(x), b(t, x)\rangle|$ are uniformly bounded, we conclude that the functions

$$
v_{j}(t):=\int_{\mathbb{R}^{d}}\left[a_{j}^{i k} \partial_{x_{i}} \partial_{x_{k}} \varphi+b^{i} \partial_{x_{i}} \varphi\right] d \mu_{t}^{j}
$$


are uniformly bounded in $t \in[0,1)$ and $j$. Therefore, the functions $t \mapsto \int_{\mathbb{R}^{d}} \varphi d \mu_{t}^{j}$, whose generalized derivatives are the functions $v_{j}$, are uniformly Lipschitzian. Hence, given a sequence of functions $\varphi_{l} \in C_{0}^{\infty}\left(\mathbb{R}^{d}\right)$, we can choose a subsequence $j_{n}$ such that all the sequences $\int_{\mathbb{R}^{d}} \varphi_{l} d \mu_{t}^{j_{n}}, l \in \mathbb{N}$, will be convergent uniformly on $[0,1)$. Together with the uniform tightness of $\left\{\mu_{t}^{j}\right\}$ for each fixed $t$, this yields a subsequence such that, for every $t \in[0,1)$, the measures $\mu_{t}^{j_{n}}$ converge weakly to some probability measures $\mu_{t}$. Clearly, for every $\varphi \in C_{0}^{\infty}\left(\mathbb{R}^{d}\right)$, the function $\int_{\mathbb{R}^{d}} \varphi d \mu_{t}$ is Lipschitzian. Hence (1.5) is satisfied.

Let us show that for almost every $t$ the measure $\mu_{t}$ is absolutely continuous and that (1.3) is satisfied. To this end, it suffices to show that, given a closed ball $B$ in $\mathbb{R}^{d}$ and a closed interval $I \subset(0,1)$, the functions $f_{j}(t, x)$ have uniformly bounded norms in $L^{r}(I \times B)$, where $r=(d+1)^{\prime}=1+1 / d$. Indeed, then, by Fatou's theorem, for a.e. fixed $t$, one has $\liminf \left\|f_{j_{n}}(t, \cdot)\right\|_{L^{r}(B)}<\infty$, whence $\mu_{t}=f(t, x) d x$ with $f(t, \cdot) \in L^{r}(B)$ for such $t$. In addition, the sequence $f_{j_{n}}$ contains a subsequence which converges weakly in $L^{r}(B)$, hence it converges to $f$ (by the weak convergence of measures), which easily yields (1.3).

Now, the last step is to show indeed the uniform boundedness of $f_{j}$ in $L^{r}(I \times B)$. Let us take a ball $B^{\prime}$ with the same center as $B$ and the radius by 1 bigger than that of $B$. Let us also take a closed interval $I^{\prime} \subset(0,1)$ strictly containing $I$. Set $\Omega=I \times B, \Omega^{\prime}=I^{\prime} \times B^{\prime}$. We observe that for every smooth function $\varphi$ with support in the interior of $\Omega^{\prime}$, one has

$$
\int_{0}^{1} \int_{\mathbb{R}^{d}}\left[\partial_{t} \varphi+a_{j}^{i k} \partial_{x_{i}} \partial_{x_{k}} \varphi\right] d \mu_{j} d t \leq C \sup \left|\nabla_{x} \varphi\right|
$$

where $C=\sup _{\Omega^{\prime}}|b(t, x)|$. It is easily seen from the proof of Theorem 3.1 in [1] (see also [1, Corollary 3.2]) and estimate (3.7) that there is a constant $\kappa=\kappa\left(C, d, \Omega, \Omega^{\prime}, \inf _{\Omega^{\prime}} \operatorname{det} A\right) \operatorname{such}$ that $\left\|f_{j}\right\|_{L^{r}(\Omega)} \leq \kappa$.

Finally, note that in the case when the coefficients are continuous in $x$ for a.e. $t$ the reasoning is similar and even simpler, since there is no need to have local $L^{p}$-estimates.

A more general result is valid if $A$ and $b$ are continuous in $x$.

Corollary 3.4. Suppose that the functions $x \mapsto a^{i j}(t, x)$ and $x \mapsto b^{i}(t, x)$ are continuous for each $t \in[0,1)$ and are bounded on bounded sets in $[0,1) \times \mathbb{R}^{d}$. In addition, suppose that, for every fixed ball $U \subset \mathbb{R}^{d}$, the functions $x \mapsto a^{i j}(t, x), t \in[0,1)$, are equicontinuous on $U$. Assume further that for each $t \in[0,1)$, there exist nonnegative compact functions $V_{t} \in C^{2}\left(\mathbb{R}^{d}\right)$ such that

$$
\lim _{|x| \rightarrow \infty}\left[a^{i j}(t, x) \partial_{x_{i}} \partial_{x_{j}} V_{t}(x)+b^{i}(t, x) \partial_{x_{i}} V_{t}(x)\right]=-\infty .
$$

Finally, assume that there exists a nonnegative compact function $\Psi \in C^{2}\left(\mathbb{R}^{d}\right)$ and a constant $C \geq 0$ such that

$$
L \Psi \leq C .
$$

Then, for every probability measure $\nu$, there exists a family $\mu=\left(\mu_{t}\right)_{t \in[0,1)}$ of probability measures on $\mathbb{R}^{d}$ satisfying (1.3) and (1.5) such that $t \mapsto \int_{\mathbb{R}^{d}} \zeta d \mu_{t}$ is continuous on $[0,1)$ for every $\zeta \in C_{0}^{\infty}\left(\mathbb{R}^{d}\right)$.

Moreover, if $\operatorname{det} A$ is separated from zero on compact subsets in $(0,1) \times \mathbb{R}^{d}$, then the continuity of $b$ in $x$ is not needed.

Proof. The reasoning is similar to the previous corollary. First of all, according to Remark 2.5 one can choose a nonnegative compact function $\Psi \in C^{2}\left(\mathbb{R}^{d}\right)$ such that $L \Psi \leq C$ and $\Psi \in L^{1}(\nu)$. Next, by using that the functions $x \mapsto a^{i j}(t, x), t \in(0,1)$, are equicontinuous on 
every fixed ball $U \subset \mathbb{R}^{d}$, i.e., have a common (for all $\left.t \in(0,1)\right)$ modulus of continuity, one can find such approximations $A_{j}=\left(a_{n}^{i j}\right)$ of $A$ that the functions $a_{n}^{i j}(t, x)$ are smooth in $x$, $\inf _{(t, x) \in(0,1] \times U} \operatorname{det} A_{j}>0$ for every ball $U$ in $\mathbb{R}^{d}, \lim _{|x| \rightarrow \infty}\left[a_{j}^{i k} \partial_{x_{i}} \partial_{x_{k}} V_{t}(x)+b^{i}(t, x) \partial_{x_{i}} V_{t}(x)\right]=-\infty$ for each $t \in(0,1)$, and $\left[a_{j}^{i k} \partial_{x_{i}} \partial_{x_{k}} \Psi(x)+b^{i}(t, x) \partial_{x_{i}} \Psi(x)\right] \leq C+1$ for all $j$. Then the rest of the proof is the same as in the previous corollary.

Remark 3.5. (i) All the above results remain valid, of course, for any time interval $[0, T)$ in place of $[0,1)$ provided that the corresponding hypotheses are valid for that interval. It is also worth noting that in place of $\mathbb{R}^{d}$ we could consider a general complete Riemannian manifold $M$. All local statements, of course, are valid automatically, and the techniques based on Lyapunov functions are justified in a similar manner (see [2] and [4], where analogous ideas are applied to elliptic equations). In particular, construction of suitable Lyapunov on manifolds is discussed in [4].

(ii) The problem of uniqueness of our solutions is open. We recall that even in the timeindependent case with $A=\mathrm{I}$ and smooth $b$, there might be no uniqueness if there is no Lyapunov function (see [3]). Another interesting open problem concerns the behaviour of the densities $p_{t}(x)$ of the measures $\mu_{t}$ as $t \rightarrow 0$. For example, if the initial value $\mu_{0}=\nu$ has a continuous density and $b$ is locally bounded, then one can show that $p_{t}(x)$ is jointly continuous up to $t=0$. We do not know whether this is true for general $b$.

In a forthcoming paper we shall obtain some infinite dimensional extensions of the above results. Let us give a sample result. Let $X$ be a separable Hilbert space with inner product $\langle x, y\rangle$. Let $\left\{e_{n}\right\}$ be an orthonormal basis in $X$. Set $x_{i}=\left\langle x, e_{i}\right\rangle$. Let $\mathcal{F C}_{0}^{\infty}\left((0,1) \times X,\left\{e_{n}\right\}\right)$ denote the space of all functions of the form $u(t, x)=u_{0}\left(t, x_{1}, \ldots, x_{n}\right)$ with $u_{0} \in C_{0}^{\infty}((0,1) \times$ $\left.\mathbb{R}^{n}\right)$. The class of all functions $u(x)=u_{0}\left(x_{1}, \ldots, x_{n}\right)$ with $u_{0} \in C_{0}^{\infty}\left(\mathbb{R}^{n}\right)$ is denoted by $\mathcal{F C}_{0}^{\infty}\left(X,\left\{e_{n}\right\}\right)$. Suppose that we have a mapping $b:[0,1] \times X \rightarrow X$ and a mapping $A$ on $[0,1] \times X$ with values in the space of nonnegative symmetric trace class operators on $X$ such that $\left\langle b, e_{i}\right\rangle$ and $\left\langle A e_{i}, e_{j}\right\rangle$ are Borel measurable. We shall say that a family of Borel probability measures $\mu_{t}, t \in[0,1)$, solves the problem

$$
L^{*} \mu=0, \quad \mu_{0}=\nu,
$$

where $\nu$ is a given Borel probability measure on $X$ if, for every $u \in \mathcal{F C}_{0}^{\infty}\left((0,1) \times X,\left\{e_{n}\right\}\right)$ and every $v \in \mathcal{F C}_{0}^{\infty}\left(X,\left\{e_{n}\right\}\right)$, one has

$$
\begin{aligned}
& \int_{0}^{1} \int_{X}\left[\partial_{t} u(t, x)\right.+\sum_{i, j}\left\langle A(x) e_{i}, e_{j}\right\rangle \partial_{e_{i}} \partial_{e_{j}} u(t, x) \\
&\left.+\left\langle b(t, x), \nabla_{x} u(t, x)\right\rangle\right] \mu_{t}(d x) d t=0 \\
& \lim _{t \rightarrow 0} \int_{X} v(x) \mu_{t}(d x)=\int_{X} v(x) \nu(d x)
\end{aligned}
$$

where we assume also the existence of all integrals. A typical existence result which can be proved by our methods is the following.

Theorem 3.6. Assume that, for some constants $C_{0}, C_{1, n}, C_{2, n}, m_{n}$, one has for all $t$ and $n$

$$
\begin{gathered}
\lim _{|x| \rightarrow \infty}\langle b(t, x), x\rangle=-\infty, \quad\langle b(t, x), x\rangle \leq C_{0}, \\
\left|\left\langle b(t, x), e_{n}\right\rangle\right| \leq C_{1, n}+C_{2, n}|x|^{m_{n}}, \quad \operatorname{trace} A(t, x) \leq C_{0} .
\end{gathered}
$$


Assume also that, for all $n, k$, every ball $U \subset X$ and every fixed $t$, the functions $x \mapsto\left\langle b(x), e_{n}\right\rangle$ and $x \mapsto\left\langle A(t, x) e_{n}, e_{k}\right\rangle$ are continuous on $U$ with the weak topology. Then, for every Borel probability measure $\nu$ possessing all moments on $X$, there exists a family $\mu=\left(\mu_{t}\right)_{t \in[0,1)}$ of Borel probability measures $\mu_{t}, t \in[0,1)$, on $X$ such that $\mu$ solves (3.8).

The proof among other things will appear in a forthcoming paper.

Acknowledgement. This work has been supported in part by the RFBR projects 00-1599267 and 01-01-00858, the INTAS project 99-559, the DFG Grant 436 RUS 113/343/0(R), the DFG-Forschergruppe "Spectral Analysis, Asymptotic Distributions, and Stochastic Dynamics", and the research programme "Analisi e controllo di equazioni di evoluzione deterministiche e stocastiche" from the Italian "Ministero della Ricerca Scientifica e Tecnologica". Most of the work was done during very pleasant visits of the first and third authors to the Scuola Normale Superiore di Pisa and the visits of the first and second authors to the University of Bielefeld.

\section{REFERENCES}

[1] V.I. Bogachev, N.V. Krylov, M. Röckner, 'On regularity of transition probabilities and invariant measures of singular diffusions under minimal conditions', Comm. Partial Diff. Equations 26 (2001), no. 11$12,2037-2080$.

[2] V.I. Bogachev, M. Röckner, 'A generalization of Hasminskii's theorem on existence of invariant measures for locally integrable drifts', Theory Probab. Appl. 45 (2000), no. 3, 417-436.

[3] V.I. Bogachev, M. Röckner, W. Stannat, 'Uniqueness of invariant measures and essential $m$ dissipativity of diffusion operators on $L^{1}$, Infinite dimensional stochastic analysis (Amsterdam, 1999), Verh. Afd. Natuurkd. 1. Reeks. K. Ned. Akad. Wet., 52, R. Neth. Acad. Arts Sci., Amsterdam, 2000, pp. 39-54.

[4] V.I. Bogachev, M. Röckner, F.-Y. Wang, 'Elliptic equations for invariant measures on finite and infinite dimensional manifolds', J. Math. Pures Appl. 80 (2001), 177-221.

[5] P. Cattiaux, C. LÉonard, Minimization of the Kullback information of diffusion processes, Ann. Inst. H. Poincaré 30 (1994), no. 1, 83-132; Correction: ibid., 31 (1995), no. 4, 705-707.

[6] G. Da Prato, J. ZAbczyk, Ergodicity for infinite-dimensional systems, Cambridge University Press, Cambridge, 1996.

[7] E.B. DAvies, ' $L^{1}$ properties of second order elliptic operators', Bull. London Math. Soc. 17 (1985), no. $5,417-436$.

[8] A.M. Il'in, R.Z. Hasminskir, 'Asymptotic behavior of solutions of parabolic equations and an ergodic property of non-homogeneous diffusion processes', Matem. Sbornik 60 (1963), no. 3, 366-392.

[9] B. JEssen, 'On the approximation of Lebesgue integrals by Riemann sums', Ann. Math. (2) 35 (1934), $248-251$.

[10] N.V. KRYlov, 'An analytic approach to SPDEs', Stochastic partial differential equations: six perspectives, R. Carmona, B. Rozovskii, Eds.; Amer. Math. Soc.: Rhode Island, Providence, 1999, pp. 185-241.

[11] O.A. Ladyz'enskaya, V.A. Solonnikov, N.N. Ural'tseva, Linear and quasilinear equations of parabolic type, Amer. Math. Soc., Rhode Island, 1968.

[12] G.M. Lieberman, Second order parabolic differential equations, World Scientific, Singapore, 1996.

[13] V. Liskevich, 'On $C_{0}$-semigroups generated by elliptic second order differential expressions on $L^{p}$ spaces', Diff. Integral Eq. 9 (1996), no. 4, 811-826.

[14] W. Stannat, '(Nonsymmetric) Dirichlet operators on $L^{1}$ : existence, uniqueness and associated Markov processes', Annali Scuola Normale Super. di Pisa Cl. Sci. (4) 28 (1999), no. 1, 99-140.

[15] W. Stannat, Time-dependent diffusion operators on $L^{1}$, Preprint SFB 343, Univ. Bielefeld, N 00-080 (2000), $41 \mathrm{pp}$. 
Department of Mechanics and Mathematics, Moscow State University, 119899 Moscow, RUSSIA

E-mail address: vbogach@mech.math.msu.su

Scuola Normale Superiore di Pisa, Piazza dei Cavalieri 7, I-56126 Pisa, Italy

E-mail address: daprato@sns.it

Fakultät für Mathematik, Universität Bielefeld, D-33501 Bielefeld, Germany

E-mail address: roeckner@mathematik. uni-bielefeld.de 